\title{
Highlights
}

\section{Using diverging predictions from classical and quantum models to dissociate between categorization systems}

Gunnar P. Epping, Jerome R. Busemeyer

- Linking classical and quantum probability theory to categorization decisions

- Novel probabilistic dissociations between two theorized categorization systems

- Comparison of Markov and quantum random walk models fit to multi-valued rating scales 


\title{
Using diverging predictions from classical and quantum models to dissociate between categorization systems
}

\author{
Gunnar P. Epping $^{\mathrm{a}, 1, *}$, Jerome R. Busemeyer ${ }^{\mathrm{a}, 1}$ \\ ${ }^{a}$ Psychological and Brain Sciences, 1101 E 10th St, Bloomington, 47405, Indiana, USA
}

\begin{abstract}
Quantum probability theory has successfully provided accurate descriptions of behavior in the areas of judgment and decision making, and here we apply the same principles to two category learning tasks, one task using informationintegration categories and the other using rule-based categories. Since information-integration categories lack verbalizable descriptions, unlike rule-based ones, we assert that an information-integration categorization decision results from an intuitive probabilistic reasoning system characterized by quantum probability theory, whereas a rule-based categorization decision results from a logical, rational probabilistic reasoning system characterized classical probability theory. In our experiment, participants learn to categorize simple, visual stimuli as members of either category $\mathrm{S}$ or category $\mathrm{K}$ during an acquisition phase, and then rate the likelihood on a scale of 0 to 5 that a stimulus belongs to one category and subsequently perform the same likelihood rating for the other category during a transfer phase. Following the principle of complementarity in quantum theory, we expect the category likelihood ratings to exhibit order effects in the information-integration task, but not in the rule-based task. In the information-integration task, we found definitive order effects in the likelihood ratings. But, in the rule-based task, we found that the order effects in the likelihood ratings are not significant.
\end{abstract}

Keywords: quantum probability theory, Markov random walk, quantum random walk, COVIS, categorization, order effects

\section{Introduction}

Although previous work applying quantum probability theory to modeling cognitive systems has provided accurate accounts of human behavior, nowhere has it been predicted when people will behave classically as opposed to when people will behave quantum-like. For the first time, we differentiate between two, analogous tasks where we predict behavior will be characterized by classical probability theory in one and by quantum probability theory in the other. COVIS, a neurobiological theory of multiple systems of category learning, has led to the discovery of over 25 behavioral and neural dissociations between rule-based (RB) and information-integration (II) category learning tasks (Ashby et al., 1998; Ashby \& Valentin, 2017). We intend to augment this literature with a probabilistic dissociation, which follows from the contrasting predictions made by classical and quantum probability theory. A hallmark divergence of quantum theory from classical theory is the noncommuntativity of sequential measurements of incompatible observables. In this article, we use this divergence to differentiate between the two theorized category learning systems.

\subsection{COVIS}

Over the last 25 years, it has been argued that two distinct systems, a declarative system and a procedural system, are recruited to learn two different types of category learning tasks, RB tasks and II tasks, respectively (Ashby \& Maddox, 2005, 2011; Poldrack et al., 2001). As postulated by COVIS, these two processes are theorized to compete

\footnotetext{
*Corresponding author. gunnarepping@gmail.com

${ }^{1}$ Declarations of interest: None 
with one another for neural resources (Ashby et al., 1998; Ashby \& Valentin, 2017; Cantwell et al., 2015). The declarative system is mediated by the prefrontal cortex, as it maintains a verbalizable rule in working memory and utilizes executive attention to observe each stimulus dimension. The procedural system is mediated by the basal ganglia, as it relies on dopamine-mediated reinforcement learning to build stimulus-response associations with the help of feedback after every trial.

In an RB task, the optimal categorization strategy is some verbalizable rule, or combination of rules, that can be combined using Boolean algebra (Ashby et al., 1998). When learning to categorize stimuli during an RB task, COVIS hypothesizes that people learn a decision bound between two categories which can be generalized across all parameter space (Casale et al., 2012). Since the learned decision bound is verbalizable and rules are logically combined, RB categorization is an explicit, analytical process.

In an II task, the optimal categorization strategy requires information from multiple stimulus dimensions to be integrated at a predecisional stage and there is no verbalizable rule to optimally categorize stimuli (Ashby \& Gott, 1988; Ashby et al., 1998). When learning to categorize during an II task, COVIS hypothesizes that people learn each category's structure in parameter space rather than learning a generalizable decision bound (Casale et al., 2012). Since the categorization strategy is not verbalizable, II categorization is an implicit, intuitive process. As indicated in Figure 1 , an RB task can be converted into an II task, and vice-versa, just by rotating the parameter space by 45 degrees.

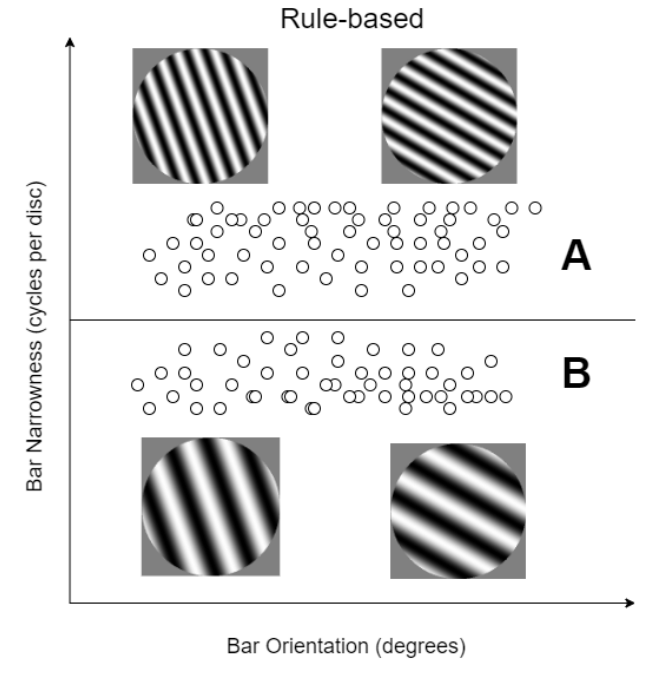

(a) RB categorization task.

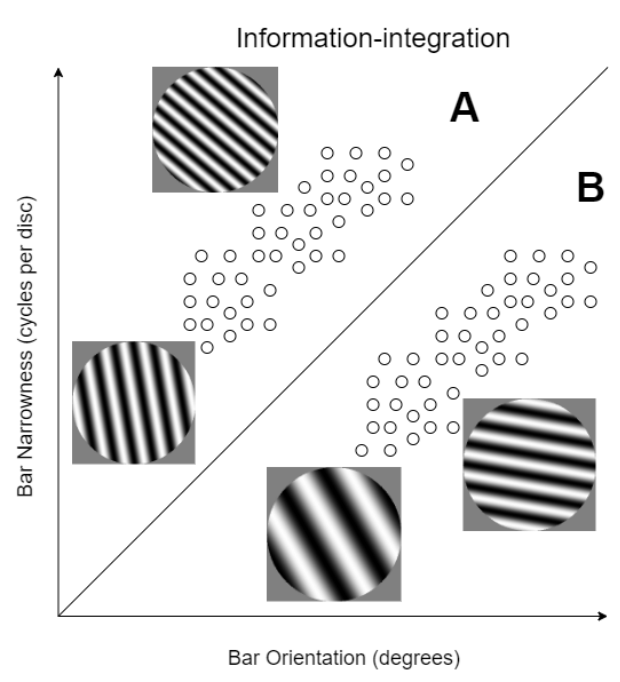

(b) II categorization task.

Figure 1: RB versus II categorization tasks.

The most prominent, alternative hypothesis to COVIS is a single system approach. The single system approach claims that many of the dissociations between RB and II tasks are confounded by differences in task difficulty (II tasks are inherently more challenging than RB ones), complexity (oftentimes, RB tasks only require selective attention to one stimulus dimension, whereas II tasks require attention to two stimulus dimensions), and/or other methodological issues (e.g., underpowered studies). Several studies have demonstrated that when these confounds are removed, the dissociations between RB and II tasks disappear (Edmunds et al., 2015; Le Pelley et al., 2019; Nosofsky et al., 2005; Zaki \& Kleinschmidt, 2014; Nosofsky \& Kruschke, 2002). Therefore, the empirical dissociations between tasks are robust, but the interpretation of what causes these dissociations (single system versus multiple systems) is being disputed. Most recently, Ashby et al. (2020) derived two novel predictions to differentiate between the multiple systems and single system hypotheses and tested these predictions on an array of categorization data. They reported that the results strongly supported the multiple systems approach over the single system one. In this article, we distinguish between the two theorized systems using predictions that follow from diverging probability theories, traditional and quantum theory, instead of using the predictions that follow from a neurobiological theory.

Part of our motivation for focusing on these theorized categorization systems is that they appear to employ different 
probabilistic reasoning systems (i.e., they handle uncertainty using different logical structures). One of the most effective ways to study probabilistic reasoning in a category learning experiment is to alter the task at some point, such that some knowledge acquired during the first component of the task must be extrapolated and applied to successfully complete the second component. Helie \& Ashby (2012) studied the effect of transferring knowledge from a direct classification task to a same-different categorization task. They found that people could only successfully apply their knowledge from the direct classification task to the same-different task when the rule that separates categories is easily verbalizable. A verbalizable rule is required for declarative, RB categorization. Without one, COVIS predicts that people will learn categories via the procedural system that underlies II categorization. Therefore, since the ability to resolve uncertainty is dependent upon a verbalizable rule, which is a unique and essential component of the RB system, this study suggests that people reach their categorization decision using different probabilistic reasoning systems to resolve uncertainty in each task. The declarative, RB system appears to use a logical reasoning system to explicitly combine measurements of different stimulus dimensions, whereas the procedural, II system appears to use a more intuitive reasoning system where measurements of different stimulus dimensions are implicitly integrated at a predecisional stage.

The other part of our motivation for focusing on these two systems is that people who utilize a more analytical style of thinking deviate from normative behavior (e.g., exhibiting order effects in judgment) to a lesser degree compared to people who utilize a more intuitive style of thinking (Yearsley \& Trueblood, 2018). We wanted to determine if the connection between style of thinking and normative behavior is merely correlational, or if the style of thinking has a causal influence on normative behavior. Since RB and II tasks provide a way to induce different styles of thinking (different probabilistic reasoning systems), we can test whether there is a causal relationship by evaluating whether the type of task has an influence on deviations from normative behavior.

\subsection{Traditional versus quantum probability theory}

In a variety of areas in psychology, models developed using the framework of quantum probability theory have accurately and coherently provided descriptions of puzzling phenomena that are problematic for traditional models (Khrennikov, 2010; Aerts, 2009; Pothos \& Busemeyer, 2022). For example, quantum models have provided explanations for violations of the sure thing principle (Pothos \& Busemeyer, 2009; Savage, 1972), conjunction and disjunction fallacies (Busemeyer et al., 2011), asymmetrical similarity judgments (Pothos et al., 2013), and order effects on inference (Trueblood \& Busemeyer, 2011). Currently, the most commonly accepted theories of probabilistic reasoning are developed using the foundation of classical theory (Oaksford \& Chater, 2007; Griffiths et al., 2010; Tenenbaum et al., 2011), which relies on Boolean logic to reduce uncertainty. This classical view of probabilistic reasoning appears to align well with RB categorization, as people are theorized to use combinations of explicit rules to make categorization decisions. Furthermore, Feldman (2000) argued that the Boolean complexity of the optimal strategy in an RB task serves as a predictor of that task's difficulty relative to other RB tasks. Given that people who employ a more intuitive style of thinking deviate from normative behavior more frequently compared to those that employ a more logical one, and that II tasks induce a more intuitive style of thinking compared to RB tasks, it is unlikely that the implicit use of category structure follows a similar line of reasoning. This is an empirical question that we investigate in this study. We suggest that quantum probability theory provides a more accurate foundation upon which to characterize probabilistic reasoning during II categorization.

Although there are several differences between traditional and quantum probability theory, only the most pertinent ones are outlined here. Classical probability theory uses subsets of a universal set to represent events (Kolmogorov, 1950), whereas quantum probability theory uses subspaces of a Hilbert space to represent events (Von Neumann, 1955). In classical theory, all variables can be measured within a single sample space, that sample space being the universal set. According to the principle of complementarity in quantum theory, quantities, or observables, can only be measured within a single sample space if they can be measured simultaneously with absolute certainty (i.e., they share a basis of eigenvectors) (Bohr, 1958). Otherwise, a change of basis is required to measure the quantities, which leads to sequential measurements that are noncommutative. Observables that are measured using different bases are said to be incompatible with each other. If all observables in a system are compatible, then the quantum description of a system reduces to a classical one. Classical theory does not permit incompatible measures because classical theory is based on Boolean logic which is communitive. Therefore, the principle of complementarity distinguishes quantum from classical probability theory (Heisenberg, 1958). 
Not only has the quantum cognition research program offered parsimonious explanations of puzzling behavior as mentioned above, but also it has provided a priori predictions that have led to findings that traditional models cannot concisely explain. Namely, the QQ inequality (Wang \& Busemeyer, 2013), interference effects of choice on confidence (Kvam et al., 2015), and temporal oscillations in preference strength (Kvam et al., 2021). As an extension to the binary choice, question order effects model (Wang \& Busemeyer, 2013), Wang \& Busemeyer (2016) developed a method to investigate sequential effects obtained using a multi-valued rating scale. Here, we adopt a similar approach to extend the quantum judgment model (Busemeyer et al., 2011) from a binary choice task to one that employs a multivalued rating scale, which allows us to compare and contrast the two theorized categorization systems by testing for order effects in category likelihood ratings.

\section{Theory}

\subsection{Order effects}

To investigate diverging predictions of classical and quantum probability theory, Wang \& Busemeyer (2016) examined sequential effects on self versus other judgments using multi-valued rating scales. In our experiment, participants learn to categorize simple, visual stimuli as members of either category K or category $\mathrm{S}$ with the help of feedback during an acquisition phase. Then, during a transfer phase, participants are asked to rate the likelihood on a scale of 0 to 5 that a stimulus belongs to one category, and subsequently perform the same likelihood rating for the other category. We investigate whether the order of the ratings has a significant effect on the joint distributions of likelihood ratings, both in an RB task and an II task.

In terms of quantum theory, both stimulus features and category likelihoods are observables. In RB categorization, verbalizable rules allow category likelihoods to be measured in terms of the stimulus features. Therefore, measurements of the stimulus features and category likelihoods take place using the same basis. Because each category likelihood is measured using the same basis as the stimulus features, all category likelihoods are measured on the same basis and they are compatible with one another. Since a change of basis is not required, we hypothesize that sequential measurements of category likelihood are commutative. Therefore, in RB categorization, we predict that there will not be order effects in category likelihood ratings.

Unlike in RB categorization, there is no explicit connection between stimulus features and category likelihood in II categorization. Therefore, it is not guaranteed that the different category likelihoods are measured using the same basis, potentially causing them to be incompatible with each other. The change of basis between category likelihood measurements could lead to sequential measurements that are noncommutative. It is important to keep in mind that when any two categories are mutually exclusive, they can be learned with absolute certainty (barring the presence of perceptual or criterial noise). Recall, when two observables (e.g., category A likelihood and category B likelihood) can be measured with absolute certainty, a quantum description of the system reduces to the classical one, and sequential measurements are expected to be commutative. It follows that when the categories of an II task are mutually exclusive and perceptual and criterial noise are negligible, we expect sequential measurements of category likelihood to be commutative. Therefore, we hypothesize sequential measurements of category likelihood are non-commutative when II categories are not mutually exclusive (i.e., categories share a region in parameter space or an exemplar) and we predict that there will be order effects in category likelihood ratings ${ }^{2}$. Note, in RB categorization, neither category overlap nor the presence of perceptual or criterial noise would have the same effect because the category likelihoods are measured in terms of stimulus features, which are compatible with one another regardless of the position of the categories in parameter space or the presence of noise.

We would like to point out that by assuming that category $\mathrm{K}$ likelihood and category S likelihoods are incompatible, we are also assuming that the questions 'Is the stimulus a member of category K?' and 'Is the stimulus not

\footnotetext{
${ }^{2}$ If category likelihoods are incompatible observables, then the two category likelihoods cannot be measured simultaneously. Therefore, during the learning phase of the II task, we hypothesize that when participants categorize a stimulus as belonging to one category, this can occur in two different ways, one for each observable. For example, if a participant decides to categorize a stimulus as a member of category K, this could occur either as a result of the participant observing that it is very likely that the stimulus belongs to category K or as a result of the participant observing that it is very unlikely that the stimulus belongs to category S. The observation of 'very likely category K' would occur as a result of measuring the stimulus on the $\mathrm{K}$ basis whereas the observation of 'very unlikely category S' would occur as a result of measuring the stimulus on the $\mathrm{S}$ basis. It is possible that participants switch between these two bases during each categorization decision, or perhaps selectively use one of the two bases that has yielded a higher accuracy up to that point.
} 
a member of category S' are not identical. Although this assumption is quite surprising and runs contrary to most people's intuition given that these questions are logically equivalent, it is important to highlight that the framing of the two questions differs. Framing effects are rampant in psychological literature. For example, framing gambles in terms of wins versus losses alters people's choice behavior (Tversky \& Kahneman, 1985) and therefore are not identical. We believe this finding could be straightforwardly extended to categorization such that framing categorization in terms of 'is a member' versus 'is not a member' alters category likelihood judgments and there are also not identical.

\subsection{Models}

We fit four different models to the joint distributions of likelihood ratings.

The first model is the rational model. The rational model does not predict order effects and assigns probabilities to category likelihoods such that $p(S)=1-p(K)$. In terms of category likelihood ratings, the rational model predicts that if the decision-maker rates the likelihood of the stimulus belonging to category $\mathrm{K}$ as 5 , then they will always rate the likelihood of the stimulus belonging to category $\mathrm{S}$ as 0 . Similarly, a rating of 4 will always be paired with a rating of 1 , and a rating of 3 will always be paired with a rating of 2. The second model is the Bayesian model. The Bayesian model does not predict order effects, but the category likelihoods are not required to sum to unity like the rational model. Because the rational model and Bayesian model do not predict order effects, we expect these two models to perform better on the RB task compared to the II task.

The third and fourth models are different types of random walk models. Sequential sampling procedures, such as random walk or diffusion processes, have proven to be effective techniques to model the dynamics of the evidence accumulation process leading up to a decision (Ratcliff et al., 2016; Link, 1975). Notably, categorization has often been viewed as an evidence accumulation process (Nosofsky \& Palmeri, 1997; Ashby, 1983; Fific et al., 2010). So far, mathematical models of these evidence accumulation processes in categorization have been employed as Markov-type sequential sampling procedures.

The third model is the Markov random walk model. Unlike the first two models, the Markov random walk model can predict order effects in category likelihood ratings. Because all measurements take place in the same sample space in a Markov random walk, the sequential effects are predicted by defining the probability of events (measurement results) in terms of both judgments and sequences of the judgments. For example, given the stimulus $\mathrm{X}$, the probability that the participant rates (judges) the likelihood of $\mathrm{X}$ belonging to category $\mathrm{K}$ to be 5 is defined as $p\left(K_{5} \mid X\right)$ and the probability that the participant rates the likelihood of $\mathrm{X}$ belonging to category $\mathrm{S}$ to be 0 is defined as $p\left(S_{0} \mid X\right)$. To define the probability in terms of sequences of judgments, the probability that the participant rates the likelihood of $\mathrm{X}$ belonging to category $\mathrm{K}$ to be 5 and then rates the likelihood of $\mathrm{X}$ belonging to category $\mathrm{S}$ to be 0 is defined as $p\left(S_{0} \mid K_{5}\right) \cdot p\left(K_{5} \mid X\right)$, whereas the probability that the participant rates the likelihood of $\mathrm{X}$ belonging to category $\mathrm{S}$ to be 0 and then rates the likelihood of $\mathrm{X}$ belonging to $\mathrm{K}$ to be 5 is defined as $p\left(K_{5} \mid S_{0}\right) \cdot p\left(S_{0} \mid X\right)$. It is not guaranteed that $p\left(S_{0} \mid K_{5}\right) \cdot p\left(K_{5} \mid X\right)=\left(K_{5} \mid S_{0}\right) \cdot p\left(S_{0} \mid X\right)$. Therefore, the Markov model can produce sequential effects by defining events in terms of sequences of judgments. Markov random walks are not the only type of random walk process, as there are also quantum random walks. Markov random walks are formulated using classical probability theory whereas quantum random walks are formulated using quantum probability theory.

The fourth model is the quantum random walk model. The quantum random walk model can also predict order effects, but it does so in a different manner compared to the Markov model. In a quantum random walk, measurements can take place in different spaces if their observables are incompatible with one another. In terms of psychology, incompatible measurements describe situations where one must take different perspectives to generate a response (Wang $\&$ Busemeyer, 2016). Since in quantum theory a measurement alters the state of the system, the first measurement sets the context for the second measurement and, as a result, the order of measurement can lead to sequential effects. Unlike the Markov random walk, the quantum model predicts sequential effects by performing different measurements on different bases. Since we predict that the measurements of category likelihood occur on one basis in the RB task and two, incompatible bases in the II task, we expect the Markov model to perform better on the RB task compared to the II task and the quantum model to perform relatively similar on both tasks ${ }^{3}$.

\footnotetext{
${ }^{3}$ Note, even though Markov models can produce order effects, they are difficult for the Markov model to adequately predict and the quantum model has been shown to predict these effects more accurately (Wang \& Busemeyer, 2016). So, these effects have turned out to be useful and diagnostic for discriminating the models.
} 


\subsection{General hypothesis and following predictions}

To summarize, we hypothesize that the probabilistic reasoning system in RB categorization is best described by classical probability theory, whereas that in II categorization is best described by quantum probability theory. We will test our hypothesis by evaluating whether the predictions that follow from it align with the empirical results on order effects and model comparison. Regarding order effects, we predict that there will not be significant order effects in the RB categorization task, but there will be significant order effects in the II categorization task. Regarding model comparison, we predict that the rational, Bayesian, and Markov models will perform better on the RB task compared to the II task and the quantum model will perform relatively similar on both tasks.

\section{Methods}

\subsection{Design}

There were a total of four experimental conditions: an RB categorization task with K-then-S transfer phase, an $\mathrm{RB}$ categorization task with S-then-K transfer phase, an II categorization task with K-then-S transfer phase, and an II categorization task with S-then-K transfer phase. Therefore, the conditions differed from one another in at least one of two aspects. Either the type of categorization task (RB or II) or the order of questioning during the transfer phase (K-then-S or S-then-K). Each subject participated in only one out of the four possible conditions. Informed consent was obtained for experimentation with human subjects.

\subsection{Participants}

In total, 153 undergraduate students from Indiana University Bloomington participated in the study. There were 38 participants in the RB and K-then-S condition, 34 participants in the RB and S-then-K condition, 49 participants in the II and K-then-S condition, and 32 participants in the II and S-then-K condition. The participants were awarded course credit for completing the study.

\subsection{Initial exclusions}

In total, 27 different participants' data sets were immediately excluded from the analysis. Participants' data sets were immediately excluded for one of the four following reasons. First, we excluded 15 participants who scored equal to or below $50 \%$ accuracy on the transfer phase because this indicated they were guessing and responding randomly. Note, since we used a 0 to 5 rating scale, a response was considered correct if either the participant responded with 0,1 , or 2 when the stimulus was not a member of the category in question or the participant responded with 3 , 4 , or 5 when the stimulus was a member of the category in question, and a response was considered incorrect if either the participant responded with 3, 4, or 5 when the stimulus was not a member of the category in question or the participant responded with 0,1 , or 2 when the stimulus not a member of the category in question. Second, we excluded eight participants who had a mean response time under 1 second because this indicated that they did not engage in a judgmental process. Third, we excluded two participants who selected the same response for the majority of their final trials because this indicated they stopped deliberating their categorization decisions as the task neared the end. Fourth, we excluded two participants who selected the same rating button for the first and second responses on approximately $80 \%$ of the transfer trials because this indicated they were not engaging in a judgemental process, at least during their second rating.

Out of the 27 that were immediately excluded, four participants were in the RB and K-then-S condition (three had a mean response time under 1 second and one gave the same response on 20 out of the last 22 trials), three participants were excluded in the RB and S-then-K condition (one scored 50\% accuracy, one had a mean response time under 1 second, and one selected the same button for both responses for 38 out of 47 trials), 15 were excluded in the II and K-then-S condition (11 scored equal to or below $50 \%$ accuracy, two had a mean response time under 1 second, one gave the same response on the final 15 trials, and one selected the same button for both responses on 40 out of 50 trials), and five were excluded in the II and S-then-K condition (three scored equal to or below $50 \%$ and two had a mean response time under 1 second). 


\subsection{Stimuli}

The stimuli are circular sine wave gratings that vary both on spatial frequency and orientation. Figure 2 is an example of one of the stimuli in the RB categorization task. The same 50 stimuli were used for each participant in the RB categorization task and the same 50 stimuli were used for each participant in the II categorization task. Each II category distribution was defined as a bivariate normal distribution, specified by a mean and variance on each dimension and by a covariance between dimensions. Each category had the same number of stimuli (25), variance on each stimulus dimension (162.5), and covariance between the two stimulus dimensions (112). To generate the RB categories, the parameter space was rotated 45 degrees counterclockwise about the mean parameters, which was 110 for both dimensions. The position of each stimulus in parameter space is depicted in Figure A.9a for the RB categorization task and in Figure A.9b for the II categorization task. The values from parameter space were used to construct sine-wave gratings of spatial frequency $x_{1}^{\prime}=0.25 \cdot x_{1}-15$ cycles $/ 100$ pixels and orientation $x_{2}^{\prime}=\pi / 180 \cdot x_{2}$ radians. This stimulus-generating procedure is similar to the one carried out in Ell \& Ashby (2006).



Figure 2: Example stimulus.

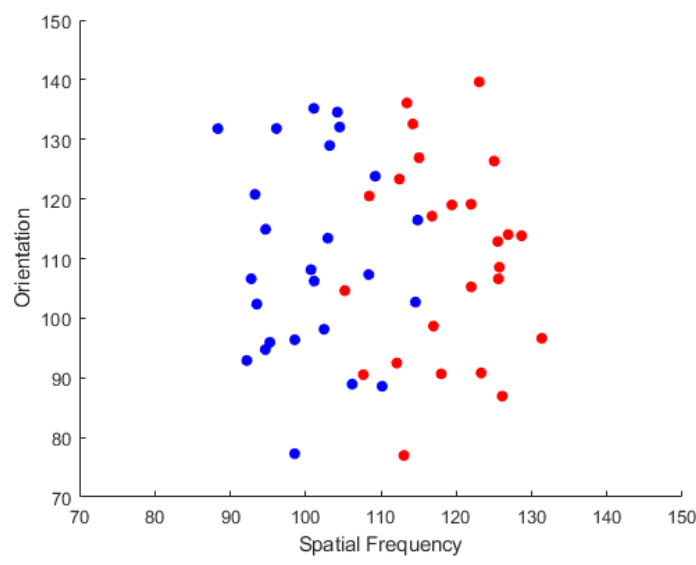

(a) RB categorization task.

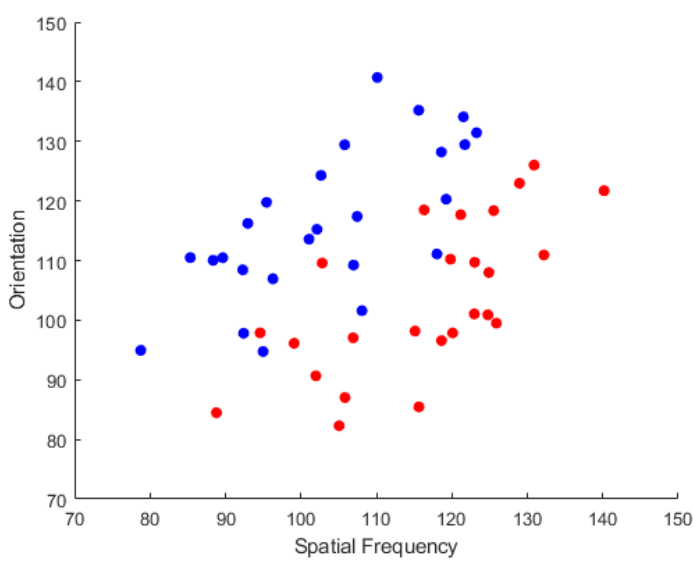

(b) II categorization task.

Figure 3: Position of each stimulus in parameter space for both tasks. The blue dots represent stimuli that are members of category $\mathrm{S}$ and the red dots represent stimuli that are members of category $\mathrm{K}$.

\subsection{Procedure}

Our experiment had two phases, a learning phase and a transfer phase. The learning phase consisted of up to 7 blocks with 50 trials per block. Each of the 50 stimuli was presented once during each block in a random order. To motivate participants, if they scored over $80 \%$ accuracy on one of the blocks, they got to skip directly to the transfer 
phase. During the learning phase of the experiment, participants learned to categorize stimuli as either a member of category S or category K, which was indicated by pressing either the " $\mathrm{S}$ " key or the " $\mathrm{K}$ " key, respectively. At first, they were guessing, but with the help of feedback, they eventually learned to properly categorize stimuli. The feedback was either "Correct" or "Incorrect", depending on the category of the stimulus and the participant's response. On each trial, a fixation cross first appeared for 1 second, and then a stimulus was presented. Once the stimulus appeared on the screen, participants had up to 5 seconds to respond with their categorization decision. If they did not respond within 5 seconds, the message "Please respond faster" appeared and the experiment skipped to the next trial.

During the transfer phase, participants completed the study in one of two question orderings: K-then-S or Sthen-K. In condition K-then-S, people would first rate the likelihood on a scale of 0 to 5 that the presented stimulus belonged to category $\mathrm{K}$ and subsequently, without altering the stimulus on the screen, rate the likelihood on a scale of 0 to 5 that the same stimulus belonged to category S. Note, a rating of 0 indicated that the stimulus is very unlikely to be a member of the category and a rating of 5 indicated that the stimulus is very likely to be a member of the category. We used a six-point scale to avoid idiosyncratic use of a 100-point scale (some people only respond with multiples of 5, others with multiples of 10, and some use the entire 100 values). The same procedure was used in the S-then-K condition, just with the order of the category likelihood ratings switched. The transfer phase consisted of 1 block with 50 trials, where each pair of ratings counted as a trial. Similar to the blocks of the learning phase, each of the 50 stimuli was presented once during the transfer block in a random order. On each trial, a fixation cross appeared for 1 second, and then the stimulus was presented. Once the stimulus appeared on the screen, participants had up to 5 seconds to respond with their likelihood rating for the first category. If they did not respond within these 5 seconds, the rating scale disappeared. After the first likelihood rating, the rating scale, but not the stimulus, disappeared for 0.1 seconds to indicate to the participants that their rating was recorded. Then, participants had up to 5 seconds to respond with their likelihood rating for the second category. If they did not respond within these 5 seconds, the message "Please respond faster" appeared and the experiment skipped to the next trial. An example of a trial in the transfer phase with the K-then-S question order is depicted in Figure 4.

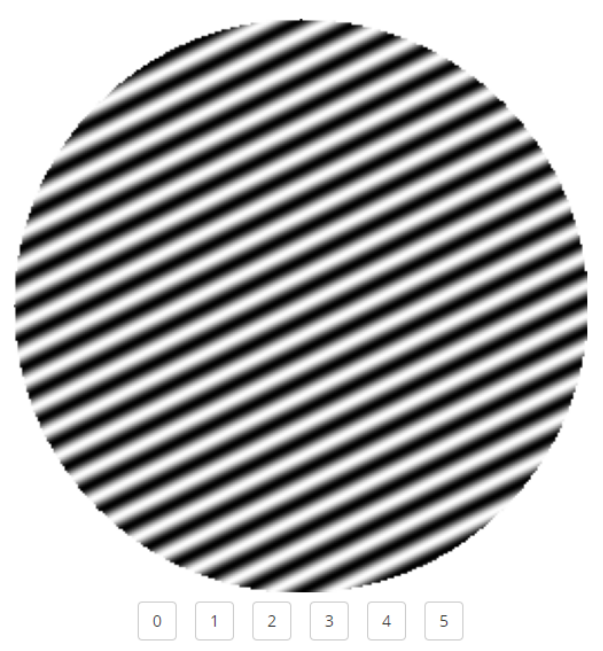

Rate the likelihood that this image belongs to category $\mathrm{K}$

(a) First, make a category $\mathrm{K}$ likelihood rating.

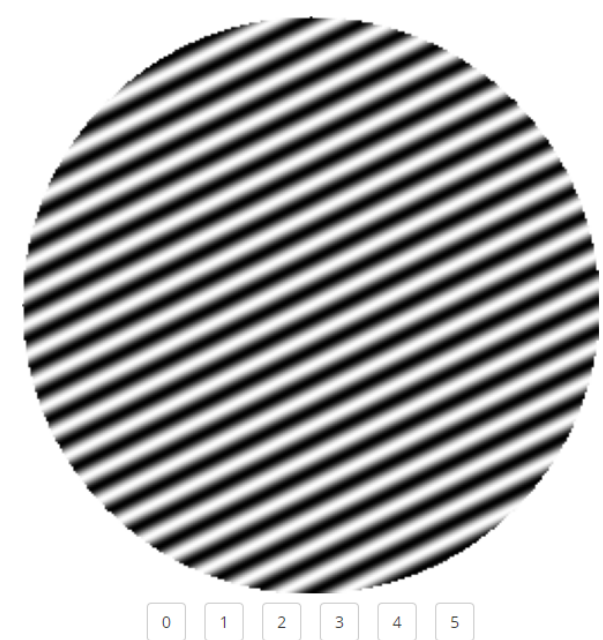

Rate the likelihood that this image belongs to category S.

(b) Then, make a category S likelihood rating.

Figure 4: Example trial with K-then-S question order.

\section{Results}

\subsection{Decision bound modeling}

Our diverging predictions on order effects are dependent on the assumption that participants adopt qualitatively different categorization strategies to categorize stimuli in each task. We assume that participants employ an explicit, declarative strategy in the RB task and a procedural strategy in the II one. To determine participants' strategies going 
into the transfer phase, we fit three different classes of models to each participant's responses during the last block of the learning phase: one class of models that assumes an explicit rule, one class that assumes a procedural strategy, and one class that assumes guessing. Note, before fitting the decision bound and guessing models, we mapped the raw coordinates of the stimuli in parameter space, $\left(x_{1}^{\prime \prime}, x_{2}^{\prime \prime}\right)$, to coordinates that more accurately correspond to people's perception of the stimuli, $\left(x_{1}, x_{2}\right)$, using a nonlinear transformation which is described in Appendix A.

We briefly outline the three classes of models here, but for more detail on each model please see Appendix B. First, two different models assume an explicit rule, the vertical decision bound (VDB) and horizontal decision bound (HDB) models. These models assume participants selectively attend to one of the two stimulus dimensions. Next, two different models assume a procedural strategy, the general linear classifier (GLC) and the general quadratic classifier (GQC). These models assume that participants integrate information from both stimulus dimensions at a predecisional stage. Lastly, two different models assume a guessing strategy, the fixed random guessing (FRG) and the general random guessing (GRG) models. The FRG assumes that each categorization decision is analogous to a coin-flip, whereas the GRG assumes that each decision is analogous to a biased coin-flip.

Each model was fit by minimizing negative log-likelihood and $B I C$ was used to compare the performance of the various models. Details for how BIC was computed for each model can be found in Appendix B. Table 1 displays the number of participants best fit by each model in each condition.

\begin{tabular}{c|cc|cc}
\hline Model & RB task, K-then-S & RB task, S-then K & II task, K-then-S & II task, S-then K \\
\hline HDB & 0 & 0 & 1 & 3 \\
VDB & 26 & 26 & 8 & 3 \\
GLC & 3 & 2 & 10 & 15 \\
GQC & 3 & 2 & 3 & 0 \\
FRG & 1 & 1 & 10 & 4 \\
GRG & 1 & 0 & 2 & 2 \\
\hline
\end{tabular}

Table 1: Number of participants best fit by each model in each condition

In the RB task, the correct rule was the VDB. Therefore, in both the K-then-S and S-then-K conditions, 26 participants used the correct RB strategy during the last block of the training phase. As a result, only the responses from these participants were included in the rest of the analysis.

In the II task, the participants that used a procedural strategy ought to have their responses best fit by the GLC or GQC. Therefore, in the K-then-S condition, it appears that 13 participants used a procedural strategy during the last block of the training phase; in the S-then-K condition, it appears that 15 participants used a procedural strategy during the last block of the training phase. As a result, only the responses from these participants were included in the rest of the analysis.

\subsection{Empirical results}

\subsubsection{Learning rates}

Figures 5 and 6 display the mean accuracy for the participants included in the analysis in each condition and the number of these participants who completed each block. To compute these learning curves, we first took each participant's accuracy on each block and then found the average and standard deviation in accuracy across participants in each block within each condition. Note, because participants got to skip to the transfer phase once they reached $80 \%$ accuracy on any training block, the average accuracy rarely goes above $80 \%$ on any block. 

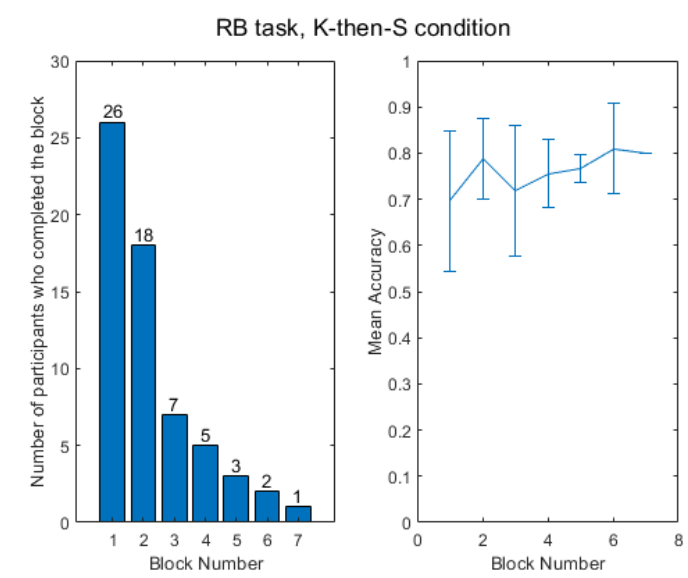

(a) Number of participants per block and mean accuracy across blocks for the K-then-S condition.

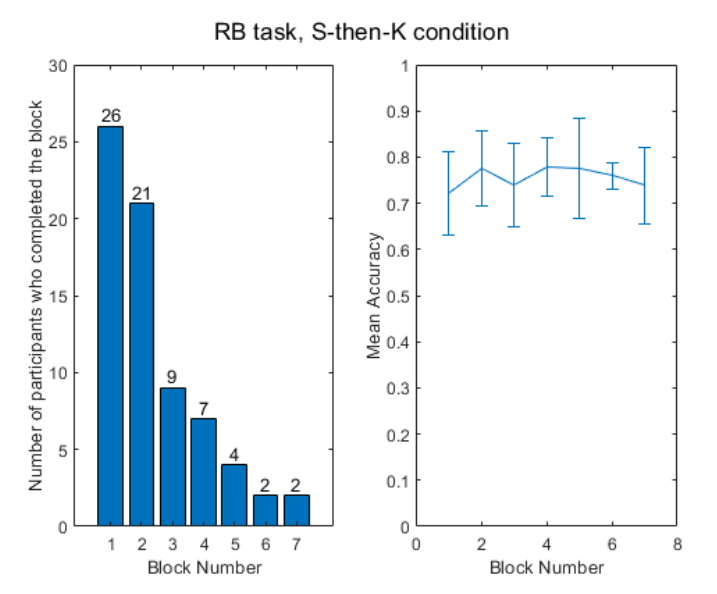

(b) Number of participants per block and mean accuracy across blocks for the S-then-K condition.

Figure 5: RB categorization task.

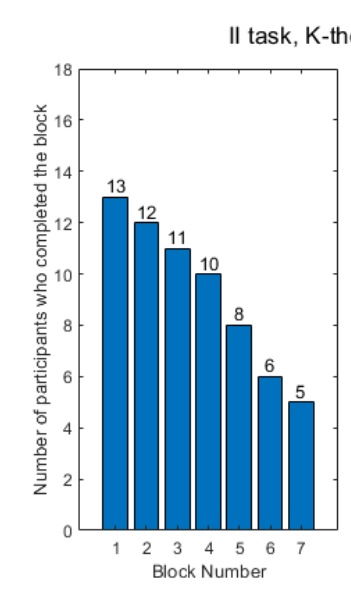

(a) Number of participants per block and mean accuracy across blocks for the K-then-S condition.

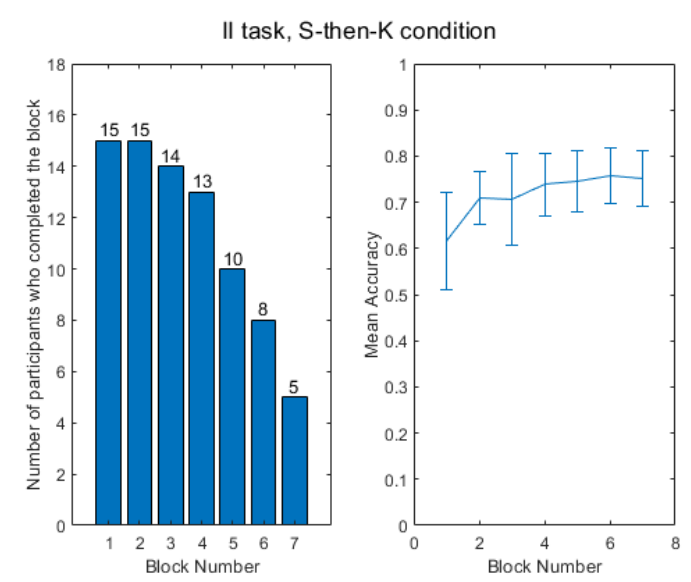

(b) Number of participants per block and mean accuracy across blocks for the $\mathrm{S}$-then-K condition.

Figure 6: II categorization task.

\subsubsection{Joint distributions}

We used two different categorization tasks (RB and II), which each have two different question orders during the transfer phase (K-the-S and S-then-K), and the joint distribution of likelihood ratings differs depending on whether a stimulus is a member of category $\mathrm{S}$ or $\mathrm{K}$. Therefore, the empirical findings from the transfer phase are depicted in eight, $6 \times 6$ tables in the Supplementary Materials (e.g., Supplementary Materials Table 1a displays the joint frequency of ratings for stimuli from category $\mathrm{K}$ when the question order was $\mathrm{K}$-then-S during the RB categorization task). Each cell in the tables indicates the relative frequency of a pair of judgments for stimuli in the specified category and question order, pooled across all stimuli in that category. In the tables, the rows correspond to the $\mathrm{K}$ ratings and the columns correspond to the $\mathrm{S}$ ratings.

Four more $6 \times 6$ tables show the differences between the relative frequencies of a pair of judgments depending on the question order (e.g., Supplementary Materials Table 1c shows the difference in the joint frequencies of ratings for $\mathrm{K}$ stimuli when category $\mathrm{K}$ likelihood is rated first compared to when category $\mathrm{K}$ likelihood is rated second). Alongside the empirical results, the predictions from the best fit random walk models are included in parentheses. 


\subsection{Tests for order effects}

To test for order effects, we computed a $\chi^{2}$-test using the four tables from each categorization task to compare two models. The $\chi^{2}$-test evaluates the significance of the difference between the $G^{2}$ statistics of the models, assuming that the observations are statistically independent. The first model is the saturated model, which does not make any assumptions. The saturated model uses four $6 \times 6$ joint distributions to fit the four tables with the constraint that they all sum to 1. For example, in the saturated model for the II categorization task, Supplementary Materials Tables 3a, 3b, $4 \mathrm{a}$, and $4 \mathrm{~b}$ are each modeled using their own joint distribution. Therefore, the saturated model has $6 \cdot 6 \cdot 4-4=140$ parameters.

The second model is the pooled-across-order model, which assumes that the same joint distribution applies to stimuli from the same category, regardless of the question order. The pooled-across-order model uses two $6 \times 6$ joint distributions to fit the four tables with the constraint that they both sum to 1 . For example, in the pooled-across-order model for the II categorization task, Supplementary Materials Tables 3a and 3b are modeled using the same joint distribution and Tables $4 \mathrm{a}$ and $4 \mathrm{~b}$ are modeled using the same joint distribution. Therefore, the pooled-across-order model has $6 \cdot 6 \cdot 2-2=70$ parameters.

\begin{tabular}{ccc}
\hline Model & $G^{2}$ & \# parameters \\
\hline Saturated & 9167.2 & 140 \\
Pooled-Across-Order & 9253.7 & 70 \\
\hline
\end{tabular}

(a) RB categorization task.

\begin{tabular}{ccc}
\hline Model & $G^{2}$ & \# parameters \\
\hline Saturated & 6208.8 & 140 \\
Pooled-Across-Order & 6323.8 & 70 \\
\hline
\end{tabular}

(b) II categorization task.

Table 2: Summary statistics from basic models for each task.

Table 2 shows the summary statistics of these models for both categorization tasks, where the statistic $G^{2}=$ $-2 \cdot \ln L($ model $)$. In the RB task, we find that with a $\Delta G^{2}=9253.7-9167.2=86.5$ and $\Delta k=140-70=70$ we cannot reject the pooled-across order model in favor of the more complex saturated model $(p=0.088>0.05)$; but, in the II task, we find that with a $\Delta G^{2}=6323.8-6208.8=115.0$ and $\Delta k=140-70=70$ we can reject the pooledacross-order model in favor of the more complex saturated model $\left(p=5.6 \cdot 10^{-4}<<0.05\right)$. Since we cannot reject the pooled-across-order model in favor of the more complex saturated model in the RB task, we can conclude that the question order did not have a significant impact on the joint distributions of the likelihood ratings during the transfer phase of the RB task. Since we can reject the pooled-across-order model in favor of the more complex saturated model in the II task, we can conclude that the question order did have a significant impact on the joint distribution of likelihood ratings during the transfer phase of the II task.

\subsection{Tests of total probability}

The 0 to 5 rating scale can be normalized by dividing it by 5 , making it comparable to the 0 to 1 probability scale. If we assume that people are directly mapping category likelihood onto the rating scale and that total probability is conserved, then $p(S)+p(K)=1$ where $p(S)$ is the normalized $\mathrm{S}$ likelihood rating and $p(K)$ is the normalized $\mathrm{K}$ likelihood rating. We would expect this negative inverse relation because $p(S)$ and $p(K)$ are mutually exclusive probabilities. By examining the proportion of normalized ratings that sum to 1 , we can test the above two assumptions.

Tables 3 and 4 show the proportion of normalized responses that sum to greater than 1, the proportion of normalized responses that sum to exactly 1 , and the proportion of normalized responses that sum to less than 1 .

In the RB task, people's responses sum to 1 on $81.68 \%$ of trials. Although the responses do not always sum to one, $81.68 \%$ is a large enough majority of responses to assume that participants are roughly mapping category likelihoods onto the rating scale and that the inverse negative relation between $p(S)$ and $p(K)$ holds in accordance with total probability.In the II task, people's responses sum to 1 on $70.31 \%$ of trials.

To evaluate whether the distribution of responses that sum to 1 is significantly different in the RB and II tasks, we first computed the proportion of responses from each participant that sum to 1, which provides us with a distribution of individual participant responses that sum to 1 in each task. Using a two-sample t-test, we found that we can reject the null hypothesis that the two distributions have equal means $(p=0.021<0.05)$ and conclude that the distribution of the individual participant responses that sum to 1 differs depending on the task. Since people appear to directly map probability onto the rating scale in the RB task and the way people utilize the rating scale should not change across 
tasks, people's responses are likely deviating from the inverse negative relation expected from mutually exclusive probabilities due to the assumption of total probability not being obeyed in the II task.

\begin{tabular}{ccc}
\hline Condition & Sum & Proportion \\
\hline \hline RB & Greater than 1 & 0.112 \\
RB & Equal to 1 & 0.847 \\
RB & Less than 1 & 0.041 \\
\hline II & Greater than 1 & 0.303 \\
II & Equal to 1 & 0.657 \\
II & Less than 1 & 0.040 \\
\hline
\end{tabular}

(a) When $\mathrm{K}$ is asked first.

\begin{tabular}{ccc}
\hline Condition & Sum & Proportion \\
\hline \hline RB & Greater than 1 & 0.118 \\
RB & Equal to 1 & 0.861 \\
RB & Less than 1 & 0.020 \\
\hline II & Greater than 1 & 0.203 \\
II & Equal to 1 & 0.780 \\
II & Less than 1 & 0.017 \\
\hline
\end{tabular}

(b) When $\mathrm{K}$ is asked second

Table 3: Sums of the joint ratings for stimuli in category $\mathrm{K}$.

\begin{tabular}{ccc}
\hline Condition & Sum & Proportion \\
\hline \hline RB & Greater than 5 & 0.147 \\
RB & Equal to 5 & 0.784 \\
RB & Less than 5 & 0.069 \\
\hline II & Greater than 5 & 0.200 \\
II & Equal to 5 & 0.766 \\
II & Less than 5 & 0.034 \\
\hline
\end{tabular}

(a) When $\mathrm{S}$ is asked first.

\begin{tabular}{ccc}
\hline Condition & Sum & Proportion \\
\hline \hline RB & Greater than 5 & 0.183 \\
RB & Equal to 5 & 0.774 \\
RB & Less than 5 & 0.044 \\
\hline II & Greater than 5 & 0.370 \\
II & Equal to 5 & 0.582 \\
II & Less than 5 & 0.047 \\
\hline
\end{tabular}

(b) When $\mathrm{S}$ is asked second.

Table 4: Sums of the joint ratings for stimuli in category S.

\subsection{Models}

Similar to Wang \& Busemeyer (2016), we defined each model on a state space formed by a lattice. Each state in the lattice corresponds to a discrete likelihood that a stimulus belongs to a category (an evidence state). The evidence states are ordered according to category likelihood, such that likelihood increases monotonically across the lattice.

When accumulating evidence during a judgment, we don't think people are limited to six evidence states (the number of different ratings on our scale) and instead believe it is more likely that people have a more refined subjective scale. We chose to use a 96-state model to more closely represent the participants' deliberation process leading up to a judgment, assign the same number of evidence states to each rating, and approximate a continuum. This is similar to the number of states used in Wang \& Busemeyer (2016) and Busemeyer \& Wang (2017) (both of these studies used a 99-state model). Additionally, we noticed that increasing the dimensionality beyond 96 states does not change the predictions of the model.

Therefore, we used a total of 96 evidence states, $\left\{\left|E_{1}\right\rangle,\left|E_{2}\right\rangle, \quad \ldots,\left|E_{95}\right\rangle,\left|E_{96}\right\rangle\right\}$ (where evidence states $16 \cdot j+1$ to $16 \cdot(j+1)$ correspond to the category likelihood rating $j$ ) so that each of the 6 ratings spans exactly 16 evidence states. The 96 evidence states form an orthonormal basis for each model.

\subsubsection{Projectors}

Six projection operators are used to pick out the probability of their respective likelihood rating. The six projection operators have zeros for all of the off-diagonal elements and the diagonal elements are (let $\overrightarrow{1} \equiv$ a vector of sixteen 1's and let $\overrightarrow{0} \equiv$ a vector of sixteen 0 's)

$$
\begin{aligned}
& P_{0}=\operatorname{diag}[\overrightarrow{1}, \overrightarrow{0}, \overrightarrow{0}, \overrightarrow{0}, \overrightarrow{0}, \overrightarrow{0}] \\
& P_{1}=\operatorname{diag}[\overrightarrow{0}, \overrightarrow{1}, \overrightarrow{0}, \overrightarrow{0}, \overrightarrow{0}, \overrightarrow{0}] \\
& P_{2}=\operatorname{diag}[\overrightarrow{0}, \overrightarrow{0}, \overrightarrow{1}, \overrightarrow{0}, \overrightarrow{0}, \overrightarrow{0}] \\
& P_{3}=\operatorname{diag}[\overrightarrow{0}, \overrightarrow{0}, \overrightarrow{0}, \overrightarrow{1}, \overrightarrow{0}, \overrightarrow{0}] \\
& P_{4}=\operatorname{diag}[\overrightarrow{0}, \overrightarrow{0}, \overrightarrow{0}, \overrightarrow{0}, \overrightarrow{1}, \overrightarrow{0}]
\end{aligned}
$$




$$
P_{5}=\operatorname{diag}[\overrightarrow{0}, \overrightarrow{0}, \overrightarrow{0}, \overrightarrow{0}, \overrightarrow{0}, \overrightarrow{1}]
$$

\subsubsection{Rational model}

For the rational model, the state of the system, $|\phi\rangle$, is represented by a linear combination of the basis states.

$$
|\phi\rangle=\sum_{j=1}^{96} a_{j} \cdot\left|E_{j}\right\rangle
$$

The coefficient of a given state is the probability that the system is in that state. Therefore,

$$
\sum_{j=1}^{96} a_{j}=1
$$

A vector of all the probabilities is the probability distribution across the evidence states. The rational model will employ two different initial states, one for category $\mathrm{K}$ likelihood ratings $\left(\left|\phi_{K}\right\rangle\right)$ and one for category $\mathrm{S}$ likelihood ratings $\left(\left|\phi_{S}\right\rangle\right)$. The initial state $\left|\phi_{K}\right\rangle$ is a Gaussian distribution which is estimated using two parameters, a mean $\mu$ and a standard deviation $\sigma^{2}$. Since the distribution of likelihood ratings in the rational model is inverted for each category, $\left|\phi_{K}\right\rangle$ and $\left|\phi_{S}\right\rangle$ are related such that

$$
\left|\phi_{S}\right\rangle=R \cdot\left|\phi_{K}\right\rangle
$$

where $R$ is a matrix with 1's on its antidiagonal and 0's elsewhere

$$
R=\left[\begin{array}{ccccc}
0 & \ldots & 0 & 0 & 1 \\
\vdots & \vdots & 0 & 1 & 0 \\
0 & 0 & & 0 & \vdots \\
0 & 1 & 0 & \vdots & \vdots \\
1 & 0 & 0 & \ldots & 0
\end{array}\right]
$$

This $R$ matrix is also used to transition between likelihood ratings on categories $\mathrm{S}$ and $\mathrm{K}$ because the probability distribution for one category is equal to the inverted probability distribution for the other category in the rational model. The joint probability for each pair of evidence ratings $i, j$ when $\mathrm{K}$ is asked first is

$$
p(\text { rating } 1=i, \text { rating } 2=j)=\| P_{j} \cdot R \cdot P_{i} \cdot\left|\phi_{K}\right\rangle \|
$$

And similarly, joint probability for each pair of evidence ratings $i, j$ when $\mathrm{S}$ is asked first is

$$
p(\operatorname{rating} 1=i, \operatorname{rating} 2=j)=\| P_{j} \cdot R \cdot P_{i} \cdot\left|\phi_{S}\right\rangle\|\Longrightarrow p(\operatorname{rating} 1=i, \operatorname{rating} 2=j)=\| P_{j} \cdot R \cdot P_{i} \cdot R \cdot\left|\phi_{K}\right\rangle \|
$$

Since we expect the initial state $\left|\phi_{K}\right\rangle$ to be different depending on whether the stimulus belongs to category S or $\mathrm{K}$, there will be two different initial states (one for a stimulus belonging to category $\mathrm{S}$ and another for a stimulus belonging to category K). Since each initial state is estimated using 2 free parameters, the rational model has a total of 4 free parameters.

\subsubsection{Bayesian model}

Similar to the rational model, in the Bayesian model, $|\phi\rangle$ is represented by a linear combination of basis states where the coefficient of a given state is the probability that the system is in that state. The Bayesian model also employs two different initial states, one for category $\mathrm{K}$ likelihood ratings $\left(\left|\phi_{K}\right\rangle\right)$ and one for category $\mathrm{S}$ likelihood ratings $\left(\left|\phi_{S}\right\rangle\right)$. Again, each initial state is a Gaussian distribution which is estimated using two parameters, a mean $\mu$ and a standard deviation $\sigma^{2}$.

The joint probability for each pair of evidence ratings $i, j$ when $\mathrm{K}$ is asked first is

$$
p(\text { rating } 1=i, \text { rating } 2=j)=\| P_{j} \cdot T_{S K} \cdot P_{i} \cdot\left|\phi_{K}\right\rangle \|
$$


and similarly, joint probability for each pair of evidence ratings $i, j$ when $\mathrm{S}$ is asked first is

$$
p(\text { rating } 1=i, \text { rating } 2=j)=\| P_{j} \cdot T_{K S} \cdot P_{i} \cdot\left|\phi_{S}\right\rangle \|
$$

where $T_{K S}$ and $T_{S K}$ are the transition operators for transitioning from $\mathrm{K}$ likelihood ratings to $\mathrm{S}$ likelihood ratings and transitioning from $\mathrm{S}$ likelihood ratings to $\mathrm{K}$ likelihood ratings, respectively. According to classical theory, the transition operator is derived from the intensity matrix of the system by solving the Kolmogorov forward equation

$$
T=\exp (\omega \cdot K)
$$

where $K$ is the intensity matrix and $\omega$ is a scaling parameter. The intensity matrix is a tridiagonal matrix that is estimated using two free parameters, a drift rate and a diffusion rate. The intensity matrix is defined as

$$
K=\left[\begin{array}{ccccc}
-c & v & 0 & \ldots & 0 \\
c & -\lambda & \ddots & \ddots & \vdots \\
0 & & \ddots & v & 0 \\
\vdots & \ddots & \ddots & -\lambda & v \\
0 & \ldots & 0 & c & -v
\end{array}\right]
$$

where without loss of generality the lower off-diagonal elements are set equal to some constant for fitting purposes $(c=1)^{4}, \lambda=v+1$, and $v>0$ is a free parameter that determines the direction of drift. If $v>c$, the distribution flows to lower evidence states, if $v<c$, the distribution flows to upper evidence states.

Because the Bayesian model must obey Bayes theorem (which causes it to not predict order effects),

$$
p(K=j \mid S=m)=\frac{p(K=j) \cdot p(S=m \mid K=j)}{p(S=m)}, p(S=m \mid K=j)=\frac{p(S=m) \cdot p(K=j \mid S=m)}{p(K=j)}
$$

must be true. Since $T_{K S}(j, m)=p(K=j \mid S=m), T_{S K}(m, j)=p(S=m \mid K=j), p(K=j)=\left|\left\langle j \mid \phi_{K}\right\rangle\right|$, and $p(S=m)=$ $\left|\left\langle m \mid \phi_{S}\right\rangle\right|$ (where $\langle j|$ and $\langle m|$ are row vectors with 1 at index $j$ and $m$, respectively, and 0's everywhere else), Bayes theorem can be rewritten as

$$
T_{K S}(j, m)=\frac{\left|\left\langle j \mid \phi_{K}\right\rangle\right| \cdot T_{S K}(m, j)}{\left|\left\langle m \mid \phi_{S}\right\rangle\right|}, T_{S K}(m, j)=\frac{\left|\left\langle m \mid \phi_{S}\right\rangle\right| \cdot T_{K S}(j, m)}{\left|\left\langle j \mid \phi_{K}\right\rangle\right|}
$$

and because $\left\langle m \mid \phi_{S}\right\rangle=\left\langle m\left|\cdot T_{S K}(m, j) \cdot\right| \phi_{S}\right\rangle(p(S=m)=p(S=m \mid K=j) \cdot p(K=j))$ and $\left\langle j \mid \phi_{K}\right\rangle=\langle j| \cdot T_{K S}(j, m) \cdot$ $\left|\phi_{K}\right\rangle(p(K=j)=p(K=j \mid S=m) \cdot p(S=m))$, it follows that

$$
T_{K S}(j, m)=\frac{\left|\left\langle j \mid \phi_{K}\right\rangle\right| \cdot T_{S K}(m, j)}{\left|\left\langle m\left|\cdot T_{S K}(m, j) \cdot\right| \phi_{S}\right\rangle\right|}, T_{S K}(m, j)=\frac{\left|\left\langle m \mid \phi_{S}\right\rangle\right| \cdot T_{K S}(j, m)}{\left|\left\langle j\left|\cdot T_{K S}(j, m) \cdot\right| \phi_{K}\right\rangle\right|}
$$

When the Bayesian model is fit to the empirical data, only one initial state and one transition matrix need to be estimated using free parameters because the other initial state and transition matrix can be defined given those two. For example, suppose we fit $\left|\phi_{K}\right\rangle$ and $T_{S K}$. Then, we could compute $\left|\phi_{S}\right\rangle$ and $T_{K S}$ from $\left|\phi_{K}\right\rangle$ and $T_{S K}$ using the following two equations

$$
\left|\phi_{S}\right\rangle=T_{S K} \cdot\left|\phi_{K}\right\rangle, T_{K S}(j, m)=\frac{\left|\left\langle j \mid \phi_{K}\right\rangle\right| \cdot T_{S K}(m, j)}{\left|\left\langle m\left|\cdot T_{S K}(m, j) \cdot\right| \phi_{S}\right\rangle\right|}
$$

\footnotetext{
${ }^{4}$ Normally, when defining the intensity matrix for a random walk process, the lower diagonal elements are set equal to a constant $\beta=\frac{\mu+\sigma}{2}$, the upper diagonal elements are set equal to $\alpha=\frac{\mu-\sigma}{2}$, and the diagonal elements are all determined such that the columns of the tridiagonal, intensity matrix all sum to 1 , where $\mu$ is the drift rate and $\sigma$ is the diffusion rate. By setting $c=1$ and using a scaling parameter, we find that $\omega=\beta=\frac{\mu+\sigma}{2}$. Similarly, here $\omega \cdot v=\alpha=\frac{\mu-\sigma}{2}$ and therefore $v=\frac{\mu-\sigma}{2} \cdot \frac{1}{\omega}=\frac{\mu-\sigma}{2} \cdot \frac{2}{\mu+\sigma}=\frac{\mu-\sigma}{\mu+\sigma}$. As you can see, we merely rearranged the parameters to make fitting the function an easier process without loss of generality.
} 
Therefore, since one initial state requires two free parameters and one transition matrix requires two free parameters (and the initial state and transition matrix will be different depending on whether the stimulus is from category $\mathrm{K}$ or category S), the Bayesian model uses a total of 8 free parameters.

\subsubsection{Markov random walk model}

For this application, we used a discreet-state, continuous-time Markov random walk process with reflecting boundaries. Again, the state of the system, $|\phi\rangle$ is represented by a linear combination of basis states where the coefficient of a given state is the probability that the system is in that state. Unlike the rational and Bayesian models, the Markov model uses one initial state, which is Gaussian distributed and defined using two free parameters (a mean $\mu$ and standard deviation $\sigma^{2}$ ).

The joint probability for each pair of evidence ratings $i, j$ is

$$
p\left(\text { rating }_{1}=i, \text { rating }_{2}=j\right)=\| P_{j} \cdot T_{2} \cdot P_{i} \cdot T_{1} \cdot|\phi\rangle \|
$$

where $T_{1}$ and $T_{2}$ are the transition operators for the first category and the second category, respectively. Since each transition matrix is estimated using two free parameters (and the transition matrices will be different depending on whether to stimulus is from category K or category S) and the initial state is estimated using two free parameters, the Markov model uses a total of 10 free parameters.

\subsubsection{Quantum random walk model}

For this application, we used a discreet-state, continuous-time quantum random walk process with reflecting boundaries. The state of the system $|\psi\rangle$ is represented by a linear combination of basis states, which here are the 96 evidence states.

$$
|\psi\rangle=\sum_{j=1}^{96} b_{j} \cdot\left|E_{j}\right\rangle
$$

The coefficient on each state is a probability amplitude which encodes the probability that the system is in that state. The probability amplitude can be converted to a probability by taking its 2-norm, or equivalently by multiplying it by its complex conjugate. Therefore,

$$
\sum_{j=1}^{96} b_{j} \cdot b_{j}^{*}=1
$$

where $b_{j}^{*}$ is the complex conjugate of $b_{j}$. A vector of all the amplitudes is the amplitude distribution across the evidence states during the evidence accumulation process. This is the first notable difference between the two different types of random walks, a Markov random walk operates on probabilities, whereas a quantum random walk operates on amplitudes.

For a quantum process, the evidence accumulation process is described by a unitary operator, which rotates the state vector in the quantum model. When applying the unitary operator to evolve the state between the two likelihood ratings, the rotation of the state could represent one of two operations. It could either represent how the state evolves due to additional information/processing time, as was assumed in Kvam et al. (2015), or it could represent the change of basis between the incompatible judgments, as was assumed in Wang \& Busemeyer (2016). Here, we assume that the rotation represents the change of basis because we felt that the II task induces incompatible representations and there is no new information presented between the two likelihood ratings. Note, the use of multiple bases versus just one basis is the second notable difference between the two random walk models.

Like the Markov model, the quantum model uses one initial state, which is Gaussian distributed and defined using two free parameters (a mean $\mu$ and standard deviation $\sigma^{2}$ ). The joint probability for each pair of evidence ratings $i, j$ is

$$
p\left(\text { rating }_{1}=i, \text { rating }_{2}=j\right)=\| P_{j} \cdot\left(U_{2} \cdot U_{1}^{\dagger}\right) \cdot P_{i} \cdot U_{1} \cdot|\psi\rangle \|^{2}
$$

where $U_{1}$ and $U_{2}$ are the unitary operators for the first category and the second category, respectively. According to quantum theory, the unitary operator is derived from the Hamiltonian matrix of the system by solving the Schrödinger equation

$$
U=\exp (-i \cdot \omega \cdot H)
$$


where $H$ is the Hamiltonian matrix, $\omega$ is a scaling parameter (similar to Planck's constant in the Schrödinger equation), and and $i$ is the imaginary number $(i=\sqrt{-1})$. Using the Schrödinger equation instead of the Kolmogorov equation to generate the dynamics of the walk is the third notable difference between quantum and Markov random walks. The Hamiltonian matrix is a tridiagonal matrix that is defined using two free parameters, a drift rate and a diffusion rate. The Hamiltonian matrix is defined as

$$
H=\left[\begin{array}{ccccc}
\mu_{1} & c & 0 & \ldots & 0 \\
c & \mu_{2} & \ddots & \ddots & \vdots \\
0 & c & \ddots & c & 0 \\
\vdots & \ddots & \ddots & \mu_{95} & c \\
0 & \ldots & 0 & c & \mu_{96}
\end{array}\right]
$$

where without loss of generality the off-diagonal elements are set equal to a constant for fitting purposes $(c=1000)$ 5 . The diagonal elements are set equal to the index of each element multiplied by the parameter $v$

$$
\mu_{j}=v \cdot \frac{j}{N}
$$

where $N$ is the number of evidence states and $v$ modulates the drift rate to diffusion rate ratio. The larger $v$ is, the stronger the influence the drift rate has on the distribution. Since each unitary matrix is estimated using two free parameters (and the unitary matrices will be different depending on whether the stimulus is from category $\mathrm{K}$ or category S) and the initial state is estimated using two free parameters, the quantum model uses a total of 10 free parameters.

\subsubsection{Non-judgmental process}

Upon further analysis, we found that participants would often skip over their judgment process on some trials and simply respond 0 and then 5 or 5 and then 0 . To account for this behavior, we included 2 additional parameters, $\alpha$ and $\beta$, to represent the probability that this non-judgmental process would take place, $\alpha$ for when category K likelihood is rated 5 and category $\mathrm{S}$ likelihood is rated 0 and $\beta$ for when category $\mathrm{K}$ likelihood is rated 0 and category S likelihood is rated 5. When including the mixture parameters, the rational model uses 6 total parameters, the Bayesian model uses 10 total parameters, and the Markov and quantum models each use 12 total free parameters.

\subsection{Model comparisons}

Two different model comparison methods were used to quantitatively compare the fits of models to the four joint distributions produced by each of the two categorization tasks. The first method estimated the parameters by minimizing the sum of squared errors $(S S E)$ between the observed frequencies and the predicted frequencies. The minimized sum of squared errors was then converted into the $R^{2}$ statistic, where $R^{2}=1-\operatorname{SSE} / T S S$ and TSS is the total sum of squared errors. The second method estimated the parameters by maximizing the log-likelihood of observed frequencies from the four tables. The log-likelihoods were then converted into the $G^{2}$ statistic, where $G^{2}=-2 \cdot \ln L($ model $)$. Note, since the rational model cannot predict responses that do not sum to unity (which as we found occurs quite frequently in the data), log-likelihood is not an appropriate way to estimate the performance of the rational model so we did not compute the $G^{2}$ statistic for the rational model. The best-fit model statistics are displayed in Table 5.

\footnotetext{
${ }^{5}$ Normally, when defining the Hamiltonian matrix for a random walk process, the off-diagonal elements are set equal to a diffusion rate $\sigma$ and the diagonal elements are determined according to some potential function. Here, we use a linear potential function, and therefore the diagonal elements are determined by the potential function $\mu_{j}=d \cdot \frac{j}{N}$ where $d$ is the drift rate of the quantum random walk. By setting $c=1000$ and using a scaling parameter, we find that $1000 \cdot \omega=\sigma$ and therefore $\omega=\frac{\sigma}{1000}$. Similarly, here $\omega \cdot \mu_{j}=\omega \cdot v \cdot \frac{j}{N}=d \cdot \frac{j}{N}$ and therefore $v=d \cdot \frac{1}{\omega}=d \cdot \frac{1000}{\sigma}=\frac{1000 \cdot d}{\sigma}$. As you can see, we merely rearranged the parameters to make fitting the function an easier process without loss of generality.
} 


\begin{tabular}{ccc}
\hline Model & RB fit & II fit \\
\hline rational & 0.909 & 0.739 \\
Bayesian & 0.851 & 0.653 \\
Markov & 0.861 & 0.669 \\
quantum & 0.948 & 0.858 \\
\hline
\end{tabular}

(a) $R^{2}$ for each model with best-fit parameters.

\begin{tabular}{ccc}
\hline Model & RB fit & II fit \\
\hline Bayesian & 11845.2 & 7741.1 \\
Markov & 11921.1 & 7810.2 \\
quantum & 10325.6 & 6741.1 \\
\hline
\end{tabular}

(b) $G^{2}$ for each model with best-fit parameters.

Table 5: Summary statistics from each model in each task.

In the RB task, the quantum model outperforms the other random walk models. The rational model also performs relatively well, providing a better fit compared to the Bayesian and Markov models. Lastly, the Bayesian and Markov models offer a similar fit.

In the II task, the quantum model again outperforms the other random walk models, but by a larger margin compared to the RB task. The rational model still outperforms the Bayesian and Markov models but does not necessarily provide a good fit to the data given that over a quarter of the variance in participants' likelihood ratings is still unaccounted for. Lastly, the Bayesian and Markov models perform similarly.

Since the quantum model performed the best in both tasks in terms of $R^{2}$, it may be more valuable to examine how the model produces the best fit. Given that the quantum model can produce classical behavior, the quantum model may be predicting joint distributions that are more indicative of classical behavior in the RB task compared to that in the II task. We tested this by comparing the magnitude of the predicted order effects across tasks, where we expect the predicted order effects in likelihood ratings to be larger in magnitude in the II task compared to that in the RB task. To do so, we used the best-fit parameters for the quantum model to obtain the predicted joint distributions of likelihood ratings in both tasks. Next, we subtracted the predicted S-then-K joint distribution from the predicted $\mathrm{K}$-then-S joint distribution (for $\mathrm{K}$ stimuli and $\mathrm{S}$ stimuli independently) to produce predicted order effect matrices. Finally, to quantify the overall predicted magnitude of order effects, we summed over the absolute value of each element in the predicted order effect matrices, and also summed across the predicted magnitude for $\mathrm{S}$ and $\mathrm{K}$ stimuli to obtain the overall predicted magnitude of order effects. We found that the predicted magnitude of order effects in the $\mathrm{RB}$ task is 1.093 , whereas that in the II task is 1.302 . Therefore, since the predicted magnitude of order effects in the II task is greater compared to that in the RB task, the best-fit quantum model produces joint distributions of responses closer to classical behavior in the RB task compared to that in the II task.

To compare the competing models while penalizing for the number of free parameters, we computed the statistics $A I C=G^{2}+2 \cdot($ \#parameters $)$ and $B I C=G^{2}+($ \#parameters $) \cdot \ln N$, where $N=2378$ in the RB categorization task ${ }^{6}$ and $N=1304$ in the II categorization task ${ }^{7}$. These statistics are displayed in Table 6 , including the saturated model from earlier as a baseline model to help evaluate the performance of the random walk models.

Note, since the quantum model provided the best fit in terms of $R^{2}$ on both tasks, the predictions from the quantum model are the ones included in parenthesis next to the empirical results included in the Supplementary Materials.

\footnotetext{
${ }^{6}$ There were 609 joint ratings for $\mathrm{K}$ stimuli in the K-then-S condition, 592 joint ratings for $\mathrm{S}$ stimuli in the $\mathrm{S}$-then-K condition, 580 joint ratings for $\mathrm{S}$ stimuli in the $\mathrm{S}$-then-K condition, and 597 joint ratings for $\mathrm{K}$ stimuli in the K-then-S condition. Therefore, $N=609+592+580+597=2378$.

${ }^{7}$ There were 297 joint ratings for $\mathrm{K}$ stimuli in the K-then-S condition, 355 joint ratings for $\mathrm{S}$ stimuli in the $\mathrm{S}$-then-K condition, 355 joint ratings for $\mathrm{S}$ stimuli in the $\mathrm{S}$-then-K condition, and 297 joint ratings for $\mathrm{K}$ stimuli in the K-then-S condition. Therefore, $N=297+355+355+297=1304$.
} 


\begin{tabular}{ccccc}
\hline Model & $G^{2}$ & \# parameters & AIC & BIC \\
\hline Saturated & 9167.2 & 140 & 9447.2 & 10255.6 \\
Bayesian & 11845.2 & 10 & 11865.2 & 11922.9 \\
Markov & 11921.1 & 12 & 11945.1 & 12014.3 \\
Quantum & 10325.6 & 12 & 10349.6 & 10418.9 \\
\hline \multicolumn{5}{c}{ (a) RB categorization task. } \\
\hline Model & $G^{2}$ & \# parameters \\
\hline Saturated & 6208.8 & 140 & 6488.8 & 7213.1 \\
Bayesian & 7741.1 & 10 & 7761.1 & 7812.8 \\
Markov & 7810.2 & 12 & 7834.2 & 7903.5 \\
Quantum & 6741.1 & 12 & 6765.1 & 6827.2 \\
\hline
\end{tabular}

(b) II categorization task.

Table 6: Model comparison statistics for both tasks.

In the RB categorization task, the quantum model outperforms the other random walk models in terms of $A I C$ and $B I C$. But, compared to the baseline saturated model, none of the random walk models offer a better fit in terms of either of those statistics. Similar to the model comparison in terms of $R^{2}$, the Bayesian and Markov models provide very similar fits to the data.

In the II categorization task, the quantum model again outperforms the other random walk models in terms of each statistic, but now it also outperforms the saturated model in terms of BIC (but not AIC). And once again, the Bayesian and Markov models offer very similar fits to the data.

\section{Discussion}

\subsection{Summary of empirical findings}

\subsubsection{Tests of order effects}

In the introduction, we indicated that the principle of complementarity distinguishes quantum from classical probability theory (Heisenberg, 1958). By hypothesizing that RB categorization measures category likelihood on the same, single basis and II categorization measures category likelihood on different bases, the principle of complementarity implies that there would be significant order effects in the joint distributions of likelihood ratings in the II task but not in the RB task when using overlapping categories. To test this, we fit a pooled-across-order model and a saturated model to the joint distributions of likelihood ratings in both tasks. By rejecting the pooled-across-order model in favor of the saturated model in the II task, but not in the RB task, we confirmed our predictions on order effects.

\subsubsection{Tests of total probability}

Since the two category probabilities are mutually exclusive and because the two probabilities sum to 1 (as required by the law of total probability), the two likelihood ratings on a given trial in the transfer phase ought to obey a strict, negative inverse relation. Therefore, the degree to which participants' joint responses conform to the negative inverse relation can be viewed as a measure of normative behavior. By using category overlap in our tasks, we expected to find that the likelihood ratings would not always sum to unity. But, we were surprised to find that, on average, participants were over $60 \%$ more likely to deviate from total probability in the II task compared to in the RB task. Although order effects were the main probabilistic dissociation we intended to demonstrate, the frequency at which participants' responses satisfied total probability is another probabilistic dissociation between RB and II categorization.

\subsubsection{Model comparison}

We used two different methods to estimate the parameters and compare the various models: $R^{2}$ and $G^{2}$. Since the individual ratings are not guaranteed to be normally distributed, the $G^{2}$ statistic provides a superior model comparison relative to the $R^{2}$ statistic. Nevertheless, the model comparison provided by the $R^{2}$ statistic is still worth noting and is required to fairly evaluate the performance of the rational model. 
First, the $R^{2}$ statistic reflects a strong divergence between the two tasks. Comparing the fits of the rational, Bayesian, and Markov models across both tasks, we find that all three of these models perform much better in the RB task compared to the II task. Although the quantum model outperforms the other random walk models in both tasks, it also performs better in the RB task compared to the II task. Looking closer at the $\Delta R^{2}$ across tasks, the quantum model accounts for about $9 \%$ less of the variance in the II task compared to that in the RB task, whereas all of the other models account for approximately $17-20 \%$ less of the variance. Therefore, the quantum model appears to be more robust, or at least provide a more similar fit to the data, across tasks compared to the other models. Using the predicted joint distributions, we found that the quantum model predicted behavior closer to classical behavior in the RB task compared to the II task. The ability of the quantum model to produce either classical or quantum behavior likely contributed to it's robustness across tasks.

The model comparison in terms of $G^{2}$ (and subsequently $A I C$ and $B I C$ ) offers a more consistent trend across the two tasks. But, we cannot directly compare these statistics across the two tasks, unlike $R^{2}$, because there is a different number of trials in each task. Therefore, it is more appropriate to compare the ordinal rank of the models in terms of fits across both tasks. In terms of both $A I C$ and BIC, the saturated model outperforms all the other models in the RB task. In the II task, the model comparison results mostly remain the same, except the quantum model outperforms the saturated model in terms of BIC. Using the saturated model as a baseline, the quantum model performs better in the II task compared to the RB one.

Taken together, these results align with our predictions that the rational, Bayesian, and Markov models perform better in the RB task compared to the II task. Although the quantum model performs better in the II task compared to the $\mathrm{RB}$ one in terms of $B I C$ relative to the saturated model, the $R^{2}$ statistic indicates the opposite result. Comparing the performance across tasks from a different angle in terms of $R^{2}$, the quantum model performs most similarly across tasks compared to the other models due to it's ability to produce either classical or quantum behavior. Therefore, the model fitting results suggest that the quantum model performs relatively similarly in both tasks, which is (albeit weakly) consistent with our predictions.

\section{Conclusion}

Unlike previous work developing quantum models of cognition, which have focused on evaluating whether behavior on a task was more quantum-like or more classical, our study applied the principle of complementarity to make an a priori prediction on behavior that would differentiate between two, analogous tasks. In doing so, we discovered a probabilistic dissociation between two categorization systems, augmenting the existing literature which is currently composed of neural and behavioral dissociations (Ashby \& Valentin, 2017). Additionally, we believe that the differences in the frequency at which total probability is satisfied also corroborate the notion that the two categorization systems employ diverging probabilistic reasoning systems (Casale et al., 2012; Helie \& Ashby, 2012). indicates the opposite result While our results from order effects and deviations from total probability support our general hypothesis, the results from the model comparison are not as definitive. In terms of $R^{2}$, although the quantum model performs the best in both tasks, the model produces more quantum-like behavior in the II task compared to the RB one. In terms of $B I C$, the quantum model outperforms the saturated model in the II task, but not the RB one.

Realistically, observations of a cognitive state are noisy (Yearsley, 2017) and thus it may be erroneous to exclusively characterize any cognitive system as exclusively quantum-like. Instead, it would be more apt to characterize cognitive systems along a continuum between classical and quantum, instead of strictly one or the other. Therefore, we conclude that altogether, the evidence at least suggests that the participants' behavior was closer to that expected by a classical probabilistic reasoning system in the RB task compared to that in the II task.

Our findings pose a new challenge to the single system approach to category learning. In the case of a single, more classical system, there would be no order effects predicted for either RB or II categorization and the classical and quantum models would be expected to perform similarly in both tasks. In the case of a single, more quantum system, there would be order effects predicted for both RB and II categorization and the quantum model would be expected to outperform the classical models by a similar margin in both tasks. These predictions from the single system approach need to be evaluated more closely, which is discussed as a future direction for research.

Besides identifying new dissociations to contribute to the single versus multiple systems debate, the results of this study also extend the work done by Yearsley \& Trueblood (2018), which identified a correlation between an intuitive 
style of thinking and deviations from rational behavior. The II categorization task induces a more intuitive style of thinking compared to that induced by the RB categorization task. Therefore, by identifying significant deviations from normative behavior in the II task and not in the RB task, we found that an intuitive style of thinking has a causal influence on deviations from rational behavior, moving beyond the previously reported correlational relationship.

\subsection{Future directions}

For future research, a relatively untouched topic is the biological implementations of quantum models of cognition. Note, the models developed under quantum cognition do not require the brain to function as a quantum computer. Rather, quantum models of cognition merely extrapolate the statistical structure of quantum mechanics and apply it to modeling cognitive systems. Busemeyer et al. (2017) developed a network of neural oscillators that is compatible with quantum cognition, as the neural oscillators evoke the phases that are produced by unitary evolution (as required by the dynamics of quantum theory). By connecting Markov and quantum random walk models to the two different systems of COVIS, we have the opportunity to investigate whether or not the neurocomputational models of COVIS can provide any insight as to what causes a neural system to produce behavior that is more quantum-like.

Another direction for future research is to alter the category structures in parameter space, such that RB and II tasks require selective attention to an equal number of stimulus dimensions. As noted in the introduction, occasionally dissociations between RB and II tasks disappear when tasks are matched in terms of complexity. Therefore, this experimental setup could provide a better way to test predictions of the single system approach.

\section{Acknowledgements}

The data reported in this study is available at https://osf.io/x2kgf/. Additionally, the code to replicate the empirical tests, fit and compare the decision bound models, and fit and compare the random walk models (as well as the best-fit parameters for each model) is available at https://osf.io/x $2 \mathrm{kgf} /$. This material is based upon work supported by the Air Force Office of Scientific Research under award number FA9550-20-1-0027. We thank Robert Nosofsky for helpful comments.

\section{References}

Aerts, D. (2009). Quantum structure in cognition. Journal of Mathematical Psychology, 53(5), 314-348.

Ashby, F. G. (1983). A biased random walk model for two choice reaction times. Journal of Mathematical Psychology, 27(3), $277-297$.

Ashby, F. G., Alfonso-Reese, L. A., Turken, A. U., \& Waldron, E. M. (1998). A neuropsychological theory of multiple systems in category learning. Psychological Review, 105(3), 442-481.

Ashby, F. G., \& Gott, R. E. (1988). Decision rules in the perception and categorization of multidimensional stimuli. Journal of Experimental Psychology: Learning, Memory, and Cognition, 14(1), 33-53.

Ashby, F. G., \& Maddox, W. T. (1993). Relations between prototype, exemplar, and decision bound models of categorization. Journal of Mathematical Psychology, 37(3), 372-400.

Ashby, F. G., \& Maddox, W. T. (2005). Human category learning. Annual Review of Psychology, 56, 149-178.

Ashby, F. G., \& Maddox, W. T. (2011). Human category learning 2.0. Annals of the New York Academy of Sciences, 1224(1), 147-161.

Ashby, F. G., Smith, J. D., \& Rosedahl, L. A. (2020). Dissociations between rule-based and information-integration categorization are not caused by differences in task difficulty. Memory Cognition, 48, 541-552.

Ashby, F. G., \& Valentin, V. V. (2017). Multiple systems of perceptual category learning: Theory and cognitive tests. In Handbook of categorization in cognitive science (pp. 157-188). Elsevier.

Bohr, N. (1958). Atomic physics and human knowledge. New York, NY: Wiley.

Busemeyer, J., \& Wang, Z. (2017). Is there a problem with quantum models of psychological measurements? PloS one, 12(11), e0187733.

Busemeyer, J. R., Fakhari, P., \& Kvam, P. (2017). Neural implementation of operations used in quantum cognition. Progress in biophysics and molecular biology, 130, 53-60.

Busemeyer, J. R., Pothos, E. M., Franco, R., \& Trueblood, J. S. (2011). A quantum theoretical explanation for probability judgment errors. Psychological Review, 118(2), 193-218.

Cantwell, G., Crossley, M. J., \& Ashby, F. G. (2015). Multiple stages of learning in perceptual categorization: evidence and neurocomputational theory. Psychonomic Bulletin Review, 22(6), 1598-1613.

Casale, M. B., Roeder, J. L., \& Ashby, F. G. (2012). Analogical transfer in perceptual categorization. Memory Cognition, 40(3), 434-449.

Crossley, M. J., Roeder, J. L., Helie, S., \& Ashby, F. G. (2018). Trial-by-trial switching between procedural and declarative categorization systems. Psychological Research, 82(2), 371-384.

Edmunds, C. E. R., Milton, F., \& Wills, A. J. (2015). Feedback can be superior to observational training for both rule-based and informationintegration category structures. The Quarterly Journal of Experimental Psychology, 68, 1203-1222. 
Ell, S. W., \& Ashby, F. G. (2006). The effects of category overlap on information-integration and rule-based category learning. Perception Psychophysics, 68(6), 1013-1026.

Feldman, J. (2000). Minimization of boolean complexity in human concept learning. Nature, 407(6804), 630-633.

Fific, M., Little, D. R., \& Nosofsky, R. M. (2010). Logical-rule models of classification response times: a synthesis of mental-architecture, random-walk, and decision-bound approaches. Psychological Review, 117(2), 309.

Griffiths, T. L., Chater, C., Kemp, C., Perfors, A., \& Tenenbaum, J. B. (2010). Probabilistic models of cognition: exploring representations and inductive biases. Trends in Cognitive Science, 14(8), 357-364.

Heisenberg, W. (1958). Physics and philosophy: The revolution in modern science, .

Helie, S., \& Ashby, F. G. (2012). Learning and transfer of category knowledge in an indirect categorization task. Psychological Research, 76(3), 292-303.

Khrennikov, A. Y. (2010). Ubiquitous quantum structure: From psychology to finance. Berlin: Springer.

Kolmogorov, A. N. (1950). Foundations of the theory of probability. New York, NY: Chelsea Publishing Co.

Kvam, P. D., Busemeyer, J. R., \& Pleskac, T. J. (2021). Temporal oscillations in preference strength provide evidence for an open system model of constructed preference. Scientific Reports, 11, 8169.

Kvam, P. D., Pleskac, T. J., \& Busemeyer, J. R. (2015). A quantum question order model supported by empirical tests of an a priori and precise prediction. Proceedings of the National Academy of Sciences of the United States of America, 112(34), 10645-10650.

Le Pelley, M. E., Newell, B. R., \& Nosofsky, R. M. (2019). Deferred feedback does not dissociate implicit and explicit category learning systems: Commentary on smith et al. (2014). Psychological Science, 30(9), 1403-1409.

Link, S. W. (1975). The relative judgment theory of two choice response time. Journal of Mathematical Psychology, 12(1), 114-135.

Nosofsky, R. M., \& Kruschke, J. K. (2002). Single-system models and interference in category learning: Commentary on waldron and ashby (2001). Psychonomic Bulletin Review, 9, 169-174.

Nosofsky, R. M., \& Palmeri, T. J. (1997). An exemplar-based random walk model of speeded classification. Psychological Review, 104(2), 266-300.

Nosofsky, R. M., Stanton, R. D., \& Zaki, S. R. (2005). Procedural interference in perceptual classification: Implicit learning or cognitive complexity? Memory Cognition, 33, 1256-1271.

Oaksford, M., \& Chater, N. (2007). Bayesian Rationality: The probabilistic approach to human reasoning. Oxford University Press.

Poldrack, R. A., Clark, J., Pare-Blagoev, E., Shohamy, D., Moyano, J. C., Myers, C., \& Gluck, M. (2001). Interactive memory systems in the human brain. Nature, 414(6863), 546-550.

Pothos, E. M., \& Busemeyer, J. R. (2009). A quantum probability explanation for violations of 'rational' decision theory. Proceeding of the Royal Society $B, 276(1665), 2171-2178$

Pothos, E. M., \& Busemeyer, J. R. (2022). Quantum cognition. Annual review of psychology, 73, 749-778.

Pothos, E. M., Busemeyer, J. R., \& Trueblood, J. S. (2013). A quantum geometric model of similarity. Psychological Review, 120(3), 679-696.

Ratcliff, R., Smith, P. L., Brown, S. D., \& McKoon, G. (2016). Diffusion decision model: Current issues and history. Trends in Cognitive Science, 20(4), 260-281.

Royston, P. (1992). Approximating the shapiro-wilk w-test for non-normality. Statistics and computing, 2(3), $117-119$.

Savage, L. J. (1972). The foundations of statistics. Courier Corporation.

Tenenbaum, J. B., Kemp, C., Griffiths, T. L., \& D., G. N. (2011). How to grow a mind: Statistics, structure, and abstraction. Science, 331(6022), 1279-1285.

Treutwein, B., Rentschler, I., \& Caelli, T. (1989). Perceptual spatial frequency - orientation surface: Psychophysics and line element theory. Perception Psychophysics, 60, 285-295.

Trueblood, J. S., \& Busemeyer, J. R. (2011). A quantum probability explanation for order effects on inference. Cognitive Science, 35(8), 15181552.

Tversky, A., \& Kahneman, D. (1985). The framing of decisions and the psychology of choice. In Behavioral decision making (pp. 25-41). Springer. Von Neumann, J. (1955). Mathematical foundations of quantum theory. Princeton, NJ: Princeton University Press.

Wang, Z., \& Busemeyer, J. (2016). Comparing quantum versus markov random walk models of judgements measured by rating scales. Philosophical Transactions of the Royal Society A: Mathematical, Physical and Engineering Sciences, 374(2058), 20150098.

Wang, Z., \& Busemeyer, J. R. (2013). A quantum question order model supported by empirical tests of an a priori and precise prediction. Topics in Cognitive Sciences, 5(4), 689-710.

Yearsley, J. M. (2017). Advanced tools and concepts for quantum cognition: A tutorial. Journal of Mathematical Psychology, 78, 24-39.

Yearsley, J. M., \& Trueblood, J. S. (2018). A quantum theory account of order effects and conjunction fallacies in political judgments. Psychonomic bulletin \& review, 25(4), 1517-1525.

Zaki, S. R., \& Kleinschmidt, D. F. (2014). Procedural memory effects in categorization: Evidence for multiple systems or task complexity? Memory Cognition, 42, 508-524.

\section{Appendix A. Transforming Parameter Space to Perceptual Space}

To construct Gabor filter stimuli similar to the ones used in this experiment, Crossley et al. (2018) first generated raw coordinates for each stimulus, $\left(y_{1}, y_{2}\right)$, from a bivariate uniform distribution on the interval $(0,100)$. To convert the raw spatial frequency coordinates, $y_{1}$, to spatial frequency values that could be used to construct the Gabor filter stimuli , $y_{1}^{\prime}$, Crossley et al. (2018) used the following nonlinear transformation defined in Treutwein et al. (1989)

$$
f\left(y_{1}\right)=2^{(3 / 100) \cdot y_{1}-1} .
$$


Therefore, in Crossley et al. (2018), the function $f()$ maps spatial frequency values in perceptual space, $y_{1}$, to spatial frequency values used to construct the Gabor filters, $y_{1}^{\prime}$.

In our study, the raw coordinates for spatial frequency, $x_{1}^{\prime \prime}$, were transformed into spatial frequency values that could be used to construct the Gabor filter stimuli, $x_{1}^{\prime}$, using the following transformation, which is similar to the stimulus generating procedure carried out in Ell \& Ashby (2006),

$$
g\left(x_{1}^{\prime \prime}\right)=\left(x_{1}^{\prime \prime} / 4-15\right) / 100 .
$$

Therefore, in our study, the function $g()$ maps raw spatial frequency values, $x_{1}^{\prime \prime}$, to the spatial frequency values used to construct the stimuli, $x_{1}^{\prime}$. The raw coordinates in parameter space and transformed coordinates in stimulus generation space for stimuli in both tasks is depicted below.

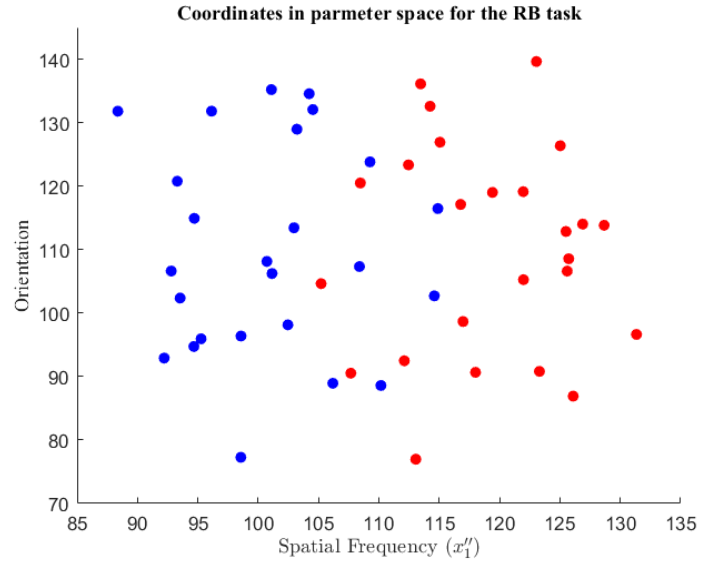

(a) RB categorization task.

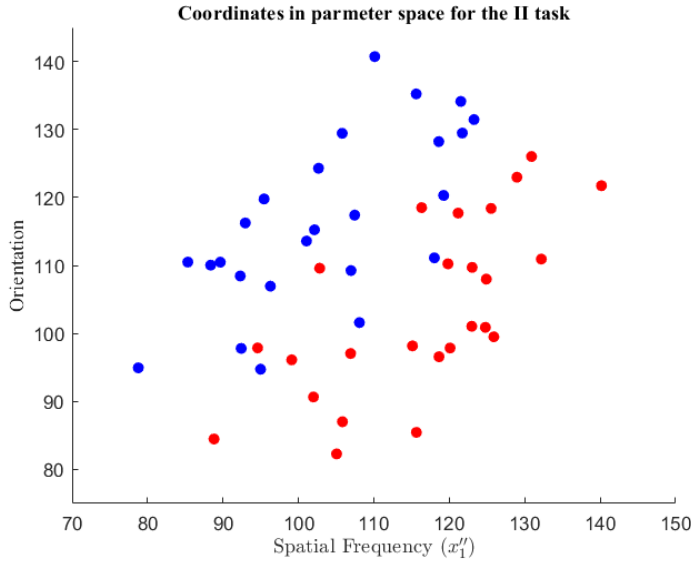

(b) II categorization task.

Figure A.7: Position of each stimulus in parameter space for both tasks. The blue dots represent stimuli that are members of category S and the red dots represent stimuli that are members of category $\mathrm{K}$.

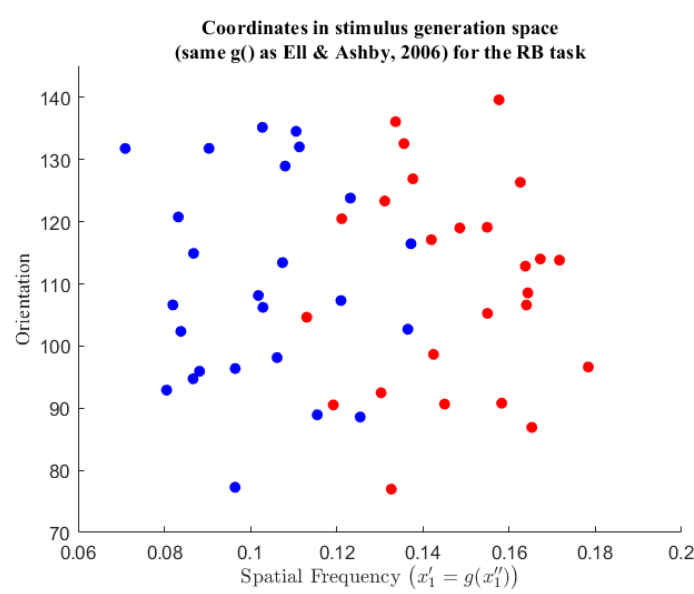

(a) RB categorization task.

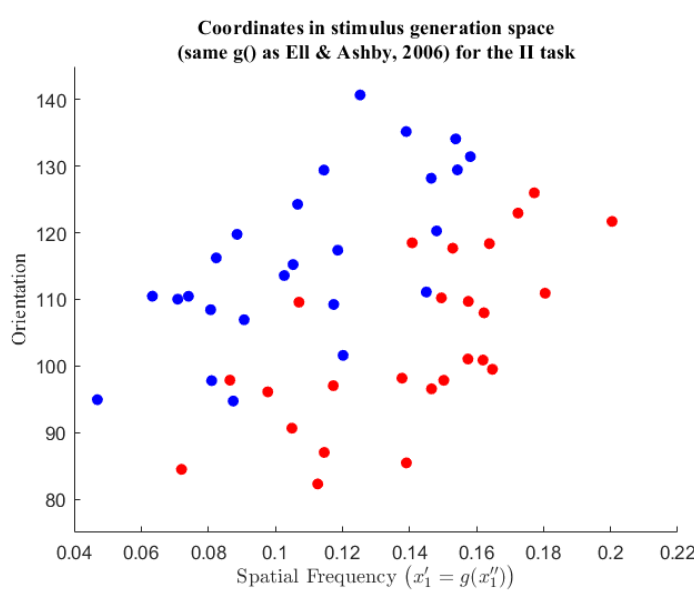

(b) II categorization task.

Figure A.8: Position of each stimulus in stimulus generating space for both tasks. The blue dots represent stimuli that are members of category S and the red dots represent stimuli that are members of category K.

To more accurately represent the position of the stimuli in perceptual space when carrying out the decision bound modeling, we used the inverse of the function employed by Crossley et al. (2018) to map the spatial frequency 
coordinates in stimulus generation space, $x_{1}^{\prime}$, into coordinates for spatial frequency in perceptual space, $x_{1}$. That is, the raw coordinates for spatial frequency in parameter space, $x_{1}^{\prime \prime}$, were first mapped by $g()$ to the spatial frequency values used to construct the Gabor filters, $x_{1}^{\prime}$, and then the spatial frequency values used to construct the Gabor filters, $x_{1}^{\prime}$, were mapped by $f^{-1}()$ to the coordinates that more accurately represent the position of the stimuli in perceptual space, $x_{1}$, where

$$
f^{-1}\left(x_{1}^{\prime}\right)=(100 / 3) \cdot\left(\log _{2}\left(x_{1}^{\prime}\right)+1\right) .
$$

Below is the explicit function used in our analysis to map the spatial frequency coordinates in parameter space, $x_{1}^{\prime \prime}$, to spatial frequency coordinates in perceptual space, $x_{1}$

$$
f^{-1}\left(g\left(x_{1}^{\prime \prime}\right)\right)=(100 / 3) \cdot\left(\log _{2}\left(g\left(x_{1}^{\prime \prime}\right)\right)+1\right)=(100 / 3) \cdot\left(\log _{2}\left(\left(x_{1}^{\prime \prime} / 4-15\right) / 100\right)+1\right) .
$$

This perceptual space for stimuli in both tasks is depicted below.



(a) RB categorization task.

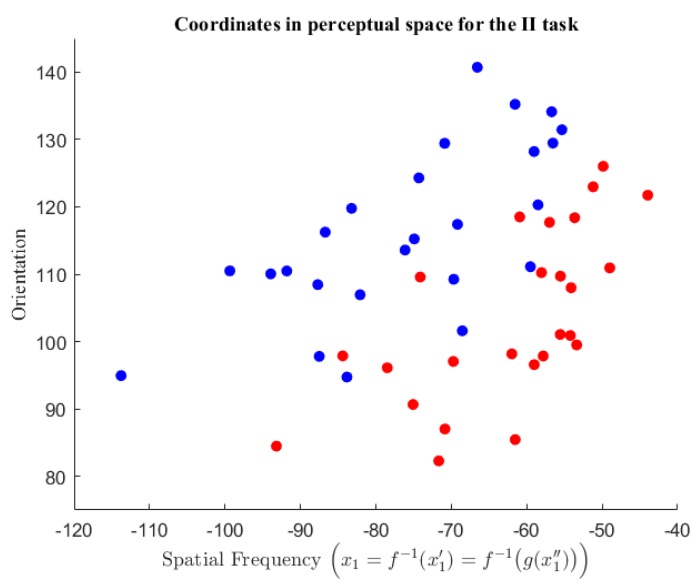

(b) II categorization task.

Figure A.9: Position of each stimulus in perceptual space for both tasks. The blue dots represent stimuli that are members of category S and the red dots represent stimuli that are members of category $\mathrm{K}$.

\section{Appendix B. Decision Bound Models}

Two different types of models that assume an explicit rule. One is the horizontal decision bound model (HDB), which assumes that participants selectively attend to the orientation of the stimuli when making categorization decisions, and the other is the vertical decision bound model (VDB), which assumes that participants selectively attend to the spatial frequency of the stimuli when making categorization decisions. The models both take the following form

$$
p\left(\text { respond } \mathrm{K} \mid S_{i}\right)=p\left(Z \leq \frac{x_{i, j}-c}{\sigma}\right),
$$

where $S_{i}$ is the stimulus, $x_{i, 1}$ is the coordinate of the spatial frequency of $S_{i}$ in perceptual space, $x_{i, 2}$ is the coordinate of the orientation of $S_{i}$ in perceptual space, $Z$ is a random variable with a standard $\mathrm{z}$ distribution, $c$ is the intercept of the decision bound, and $\sigma$ is the variance of perceptual and criterial noise. For the HDB, $j=2$; and, for the VDB, $j=1$. Both models have two free parameters $(c, \sigma)$.

Two different types of models that assume a procedural strategy. One is the general linear classifier (GLC), which assumes that participants separate the categories using a linear decision bound, and the other is the general quadratic classifier (GQC), which assumes that participants separate the categories using a quadratic decision bound. When (1) the categories are normally distributed along each dimension, (2) the variance on each stimulus dimension in one category is equal to that in the other category, and (3) the covariance between the two dimensions is the same for each category, then the optimal bound can be specified using the GLC (Ashby \& Maddox, 1993). But when the 
categories are (1) normally distributed along each dimension and either (2a) the variance on any stimulus dimension of one category is different than the same stimulus dimension in the other category or $(2 b)$ the covariance between the two dimensions is not the same for each category, then the optimal bound can be specified using the GQC (Ashby \& Maddox, 1993).

Due to the nonlinear transformation from parameter space to perceptual space, in both RB and II tasks, the variance of the spatial frequency dimension in category $\mathrm{K}$ is not equal to that in category S. Additionally, in the II task, the covariance between the two stimulus dimensions in category $\mathrm{K}$ is not equal to that in category S. Although the nonlinear mapping changes the shape of the categories in both tasks, we do not need to drop our assumption of that the stimuli are normally distributed when determining the optimal bound. We used the Shapiro-Wilk test (Royston, 1992) to evaluate whether we can reject the hypothesis that the transformed distributions of spatial frequencies in each category are sampled from a normal distribution. We found that we cannot reject the normality assumption for any of the distributions of spatial frequencies (all p-values $>0.05$ ). Granted, just because the normality hypothesis cannot be rejected does not mean that the data is normally distributed, but rather it means that we cannot reject the normality assumption. Therefore, although the variance of the spatial frequency coordinates of each category is altered when mapping the stimulus coordinates from parameter space to perceptual space, we can retain our normality assumption regarding the distribution of the stimulus coordinates of each category in each task on each dimension. Therefore, the optimal bound in both RB and II tasks is specified by the GQC (although we do not expect participants in the RB task to abandon an explicit rule in favor of a procedural one because the procedural strategy does not offer an improvement in accuracy in this study). The GQC has the following form

$$
p\left(\operatorname{respond} \mathrm{K} \mid S_{i}\right)=p\left(Z \leq \frac{a_{1} \cdot x_{i, 1}^{2}+a_{2} \cdot x_{i, 2}^{2}+a_{3} \cdot x_{i, 1} \cdot x_{i, 2}+a_{4} \cdot x_{i, 1}+a_{5} \cdot x_{i, 2}+c}{\sigma}\right),
$$

Although there appears to be seven free parameters $\left(a_{1}, a_{2}, a_{3}, a_{4}, a_{5}, c, \sigma\right)$, only six free parameters are required to fit the GQC. To account for this, we set $a_{4}=\sin (\theta)$ and $a_{5}=\cos (\theta)$. Therefore, the GQC has six free parameters $\left(a_{1}, a_{2}, a_{3}, \theta, c, \sigma\right)$. The GLC is a nested model of the GQC. By setting $a_{1}=a_{2}=a_{3}$, the GQC reduces to the GLC and therefore the GLC has three free parameters $(\theta, c, \sigma)$.

Two different types of models that assume guessing. One is the fixed random guessing model (FRG), which assumes that participants categorize stimuli as members of category $\mathrm{K}$ with $p=0.5$ and categorize stimuli as members of category $\mathrm{S}$ with $p=0.5$ (basically, each categorization decision is a coin flip), and the other is the general random guessing model (GRG), which assumes that participants categorize stimuli as members of category $\mathrm{K}$ with $p=p^{\prime}$ and categorize stimuli as members of category $\mathrm{S}$ with $p=\left(1-p^{\prime}\right)$ (here, each categorization decision is a biased coin flip). The FRG has no free parameters and the GRG has one free parameter $\left(p^{\prime}\right)$ to specify the probability of categorizing stimuli as members of category $\mathrm{K}$.

$B I C$ was used to compare the performance of the various models for each participant. The formula for BIC is

$$
B I C(\text { model })=-2 \cdot L L(\text { model })+k \cdot \log (N)
$$

where $L L$ is the log-likelihood of the model, $k$ is the number of free parameters of the model, and $N$ is the number of trials during the last block of the training phase. Note, although each block consisted of 50 trials, some participants (21 out of the 126 participants included in the decision bound modeling analysis) did not respond on every trial. The average (mean) number of trials during the last block of the learning phase across the 126 participants is 49.73 trials. 